\title{
Bifurcation Analysis of Piecewise Smooth Ecological Models
}

Fabio Dercole (dercole@elet.polimi.it)

Alessandra Gagnani (gragnani@elet.polimi.it)

Sergio Rinaldi (rinaldi@elet.polimi.it)

\section{Approved by}

Ulf Dieckmann

Leader, Evolution and Ecology Program

December 2007 


\section{Contents}

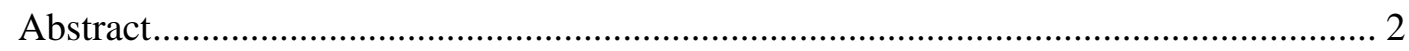

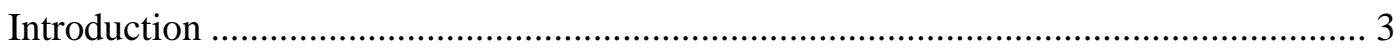

Filippov systems, sliding bifurcations and characteristic frame.................................. 7

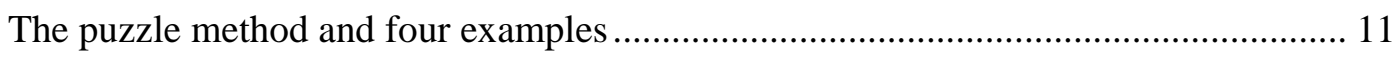

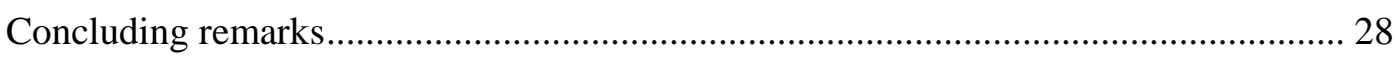

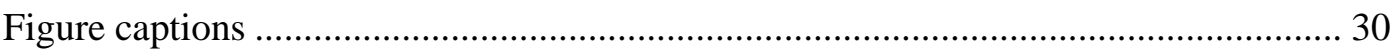

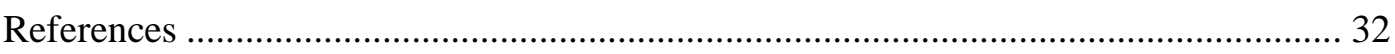

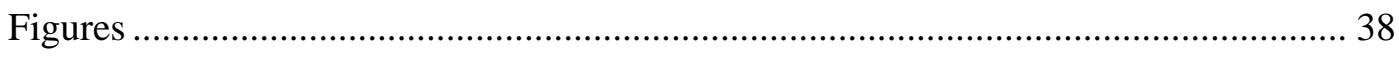




\title{
BIFURCATION ANALYSIS OF PIECEWISE SMOOTH ECOLOGICAL MODELS
}

\author{
Fabio Dercole ${ }^{1}$, Alessandra Gragnani ${ }^{1}$, Sergio Rinaldi 1,2
}

${ }^{1}$ Dipartimento di Elettronica e Informazione, Politecnico di Milano, Via Ponzio 34/5, 20133 Milano, Italy

2 Evolution and Ecology Program, International Institute for Applied Systems Analysis, A2361 Laxenburg, Austria

Corresponding author:

Fabio Dercole

Dipartimento di Elettronica e Informazione, Politecnico di Milano

Via Ponzio 34/5, 20133 Milano, Italy

Phone: $+39-02-23993484$

Fax: $\quad+39-02-23993412$

Email: dercole@elet.polimi.it

Key words: Filippov systems, sliding, renewable resources management, prey-predator systems, bifurcation analysis 


\begin{abstract}
The aim of this paper is the study of the long-term behavior of population communities described by piecewise smooth models (known as Filippov systems). Models of this kind are often used to describe populations with selective switching between alternative habitats or diets or to mimic the evolution of an exploited resource where harvesting is forbidden when the resource is below a prescribed threshold. The analysis is carried out by performing the bifurcation analysis of the model with respect to two parameters. A relatively simple method, called the puzzle method, is proposed to construct the complete bifurcation diagram step-bystep. The method is illustrated through four examples concerning the exploitation and protection of interacting populations.
\end{abstract}




\section{INTRODUCTION}

In recent years there has been an increasing interest for models in which the smooth evolution of the relevant variables is accompanied, from time to time, by abrupt changes in behavior. Plankton blooms in shallow lakes, recurrent invasions of insect pests in forests, children epidemics, pulsed chemotherapy, populations with selective switching between alternative habitats are important examples. Systems of this kind are generically called discontinuous, or hybrid, because they can be viewed as the interaction of two compartments working one in continuous-time and the other in discrete-time.

There are, however, various classes of discontinuous systems which have their own special features and require different methods for their analysis. A first class is that of "impact models" originally used in mechanics (see Brogliato, 1999 and references therein) to describe the dynamics of mechanical systems characterized by impacts among various masses. They are quite special and can be used to explain a number of rather subtle phenomen like the "Zeno chattering" (e.g., the diminishing return times of the impacts of a ping-pong bouncing ball) that other models cannot explain. Impact models represent the most naïve approach to the description of systems characterized by dynamic phenomena occurring at very diversified time scales. The first application in ecology concerns natural forests which grows smoothly and slowly but are devastated by fire from time to time (Maggi and Rinaldi, 2006). Impact models should not be confused with an apparently similar but substantially different class of models, namely that of "periodically pulsed" systems where the discontinuity in state space is generated by a periodic exogenous shock on the system. Many are the examples of this second class of discontinuous models in biology: the control of continuously stirred-tank reactors (Funasaki and Kot, 1993), the study of pulsed chemotherapy (Lakmeche and Arino, 2001) and vaccination (Shulgin et al., 1998) and a number of contributions dealing with the effects of periodic harvesting or immigation (Grant et al., 1997; Chau, 2000; Ives et al., 2000; Liu and Chen, 2003; Geritz and Kisdi, 2004; Reluga, 2004). However, in these models the return time of the discontinuous event is constant and a priori fixed, while in impact models the return times are neither constant nor pre-specified but are endogenously created by the interactions among the relevant variables of the system.

A third class of discontinuous system, which is the one we discuss in this paper, is that of "piecewise smooth" systems, often called Filippov systems, described by standard ordinary differential equations 


$$
\dot{x}(t)=f(x(t)), \quad x \in R^{n}
$$

where, however, the function $f$ is discontinuous on a number of $(n-1)$-dimensional manifolds. To our knowledge, the first formal example of a Filippov system in ecology can be found in Krrivan (1996) where the dynamics of a population of predators feading on two types of prey is studied. Under standard assumptions on optimal foraging behavior (Stephens and Krebs, 1986) the problem is reduced to the analysis of a simple third-order (i.e., $n=3$ ) Filippov system. A second example (Křivan and Sirot, 1997) deals with a host-parasitoid system where the parasitoids split their time between hosts and food searching. Again, under realistic assumptions on the individual strategy of parasitoids, the problem is reduced to the study of a second-order (i.e., $n=2$ ) Filippov system, which allows one to discuss persistence in a rather effective and compact form.

In the last ten years many other problems of population dynamics have been approached (mainly by Křivan and coauthors) through Filippov models (Křivan, 1997a, 1997b; Sirot and Křivan, 1997; Křivan, 1998; Boukal and Křivan, 1999; Genkai-Kato and Yamamura, 1999; Křivan and Sikder, 1999; Van Baalen et al., 2001; Křivan, 2003; Křivan and Eisner, 2003; Křivan and Schmitz, 2003; Křivan and Diehl, 2005; Srinivasu and Gayatri, 2005; Schreiber et al., 2006). Abstractly speaking, these problems deal with populations in which individuals are fitness-maximizers so that their optimal strategy is to switch between alternative habitats or diets as soon as the density of some population becomes too high or too low.

Filippov models could also be used to mimic the evolution of exploited populations if harvesting is forbidden when the population is below a critical threshold (Dercole et al., 2003; Meza et al., 2005; Costa and Meza, 2006). This is actually the sense of the use of quotas in fisheries (Hilborn and Walters, 1992) and is common practice in timber production in exploited forests (Davis and Johnson, 1987; Fredericksen, 1998). Similar considerations hold if, in order to avoid human epidemics, a particular fishery is forced to temporarily stop its activity as soon as the concentration of a particular contaminant in the product raises above a prespecified threshold. Finally, Filippov models have also been used in problems of evolutionary biology where selective pressures can abruptly change during the evolution of the relevant phenotypic traits (Dercole et al., 2006).

In this paper we consider the simplest class of Filippov systems, namely that of secondorder systems $(n=2)$, in which the discontinuity occurs on a curve $\Sigma$, called boundary, 
partitioning the two-dimensional state space into two open regions $S_{1}$ and $S_{2}$ in which the function $f$ is smooth. The analysis of this class of systems (Filippov, 1964; Filippov, 1988), is nontrivial since the vector $\dot{x}$ is not defined on $\Sigma$ and has two different limit values, say $f^{(1)}$ and $f^{(2)}$, in $S_{1}$ and $S_{2}$. If the components of $f^{(1)}(x)$ and $f^{(2)}(x)$ transversal to $\Sigma$ have the same sign, as in Fig. 1a, the orbit crosses the boundary $\Sigma$ and has, at that point, a discontinuity in its tangent vector. On the contrary, if the transversal components of $f^{(1)}(x)$ and $f^{(2)}(x)$ are of opposite sign, i.e. if the two vector fields are "pushing" in opposite directions, as in Fig. 1b, the state of the system is forced to remain on the boundary and slide on it. Although, in principle, motions on the boundary could be defined in different ways, the only reasonable one is Filippov convex rule (Filippov, 1964; Filippov, 1988) that defines sliding motions on $\Sigma$ as specified in the next section. Thus, the state portrait of a Filippov system is composed of the sliding state portrait on $\Sigma$ and of the standard state portraits in regions $S_{1}$ and $S_{2}$. Notice that Filippov systems can be irreversible, since the same point on $\Sigma$ can be reached through two distinct paths (see Fig. 1b).

Very often models are used to detect all qualitatively different asymptotic behaviors of a population community for all possible values of some demographic and/or environmental parameter. For example, if a harvested predator $x_{2}$ feeds selectively on a prey $x_{1}$ and on a second unpalatable but guaranteed prey, it is interesting to know how many are the different long term scenarios obtainable by varying the harvesting effort $E$. Under suitable assumptions on selectivity (Genkai-Kato and Yamamura, 1999) the problem can be modeled as a secondorder Filippov system depending upon the parameter, and the analysis shows (see Example 2 below) that there are four possible outcomes, as shown in Fig. 2 where the thick vertical line is the sliding trajectory. For low efforts (Fig. 2a) prey and predator coexist on a limit cycle which contains a sliding phase during which the predators are hesitating between their two sources of food. Higher harvesting efforts reduce prey-predator oscillations at the point of eliminating the sliding phase (Fig. 2b). For a further increase of the effort, the coexistence becomes stationary (i.e., the cycle in Fig. $2 \mathrm{~b}$ shrinks and becomes a stable equilibrium point in Fig. 2c), while for very high efforts the predator population goes extinct (Fig. 2d).

Obtaining the complete catalogue of long term behaviors of a given parameterized system is not a simple task. This is often done through the systematic analysis of a huge number of long simulations characterized by different initial conditions and parameter values. Although very popular, this approach is weak for two reasons. First, there is no guarantee that all 
possible asymptotic behaviors are detected because some of them might correspond to untested windows of initial conditions and/or parameter values. Second, and most important, the critical value of a parameter at which a change occurs in the asymptotic behavior of a dynamical system can not be obtained precisely through simulation. Indeed, these critical parameter values are nothing but the so-called bifurcations of the model (Arnold, 1982; Guckenheimer and Holmes, 1986) at which, by definition, there is a transition from stability to instability in some component of the system. Thus, when a parameter is close to its critical value the simulations must be so long for distinguishing a very weak stability from a very weak instability, that it becomes practically impossible to understand if the parameter is subcritical or supercritical. By contrast, numerical bifurcation analysis (Kuznetsov, 2004) does not suffer these limitations and is therefore the most appropriate tool for determining the complete catalogue of behaviors of a parameterized family of dynamical systems. Moreover, effective packages are nowadays available for the almost automatic bifurcation analysis of continuous dynamical systems with respect to two parameters.

Unfortunately, the situation is not as simple for Filippov systems which besides the standard bifurcations of continuous systems, can have very special bifurcations called sliding bifurcations (Feigin, 1994; di Bernardo et al., 1998a, b; di Bernardo et al., 1999; di Bernardo et al., 2001; di Bernardo et al., 2002; di Bernardo et al., 2003). However, all possible sliding bifurcations of second-order Filippov systems have recently been listed (Kuznetsov et al., 2003) using the classical approach of topological equivalence (Arnold, 1982). Consistently with this approach, all bifurcations (standard and sliding) can be interpreted as collisions among the standard and/or sliding trajectories composing a special set of trajectories, here called characteristic frame. For example, in Fig. 2 the characteristic frame is composed of the sliding segment, the cycle and the equilibria, and, indeed, the bifurcations separating the four scenarios correspond to collisions among these special trajectories. The aim of this paper is to show that the characteristic frame is a powerful tool for detecting, through a method called puzzle method, the complete catalogue of the possible asymptotic behaviors of a Filippov system.

The paper is organized as follows. In the next section we briefly review second-order Filippov systems and their sliding bifurcations, and we define the characteristic frame. Then, we describe the puzzle method through the discussion of four ecological examples which are presented in the order of increasing difficulty: the first one can be studied analytically, while the others require the use of SlideCont (Dercole and Kuznetsov, 2004; Dercole and 
Kuznetsov, 2005), a package accompanying AUTO97 (Doedel and Kernévez 1986; Doedel et al., 1997) for the numerical bifurcation analysis of second-order Filippov systems. Almost all possible sliding bifurcations of second-order Filippov systems are involved in at least one of the examples. A final section summarizes the results and points out open problems.

\section{FILIPPOV SYSTEMS, SLIDING BIFURCATIONS AND CHARACTERISTIC FRAME}

In this section we consider generic second-order (i.e. $x \in R^{2}$ ) Filippov systems described by

$$
\dot{x}= \begin{cases}f^{(1)}(x), & x \in S_{1} \\ f^{(2)}(x), & x \in S_{2}\end{cases}
$$

where the regions $S_{1}$ and $S_{2}$ are separated by the discontinuity boundary $\Sigma$ described by

$$
H(x)=0
$$

where $H$ is a smooth scalar function with nonvanishing gradient $H_{X}(x)$ on $\Sigma$.

Solutions of (1) can be constructed by concatenating standard solutions in $S_{1,2}$ and sliding solutions on $\Sigma$ obtained with the Filippov convex rule (Filippov, 1964; Filippov, 1988, Broucke et al., 2001). First we define the crossing set $\Sigma_{C} \subset \Sigma$ as the set of all points $x \in \Sigma$, where the two vectors $f^{(i)}(x)$ have nontrivial transversal components to $\Sigma$ of the same sign. By definition, at these points the orbit of (1) crosses $\Sigma$, i.e. the orbit reaching $x$ from $S_{i}$ concatenates with the orbit entering $S_{j}, j \neq i$, from $x$.

Then, we define the sliding set $\Sigma_{S}$ as the complement to $\Sigma_{C}$ in $\Sigma$. The sliding set may contain singular sliding points at which either both vectors $f^{(1)}(x)$ and $f^{(2)}(x)$ are tangent to $\Sigma$, or one of them vanishes while the other is tangent to $\Sigma$, or both vanish. 
The Filippov rule associates the following convex combination $g(x)$ of the two vectors $f^{(i)}(x)$ to each nonsingular sliding point $x \in \Sigma_{S}$ :

$$
\begin{aligned}
& g(x)=\lambda f^{(1)}(x)+(1-\lambda) f^{(2)}(x) \\
& \lambda=\frac{\left\langle H_{x}(x), f^{(2)}(x)\right\rangle}{\left\langle H_{x}(x), f^{(2)}(x)-f^{(1)}(x)\right\rangle}
\end{aligned}
$$

where $\langle\cdot$,$\rangle denotes the standard scalar product. As indicated in Fig. 3, g(x)$ is tangent to sliding segments of $\Sigma_{S}$.

Thus,

$$
\dot{x}=g(x), \quad x \in \Sigma_{s}
$$

defines a scalar differential equation on $\Sigma_{S}$, which is smooth on one-dimensional sliding intervals of $\Sigma_{S}$. Solutions of this equation are called sliding solutions.

An approximate way of solving system (1) brings to the notion of chattering solution, described in Fig. 4. In practice, an $\varepsilon$-tube is constructed around $\Sigma$ and the orbit in $S_{1}\left[S_{2}\right]$ is extended also in $S_{2}\left[S_{1}\right]$ until it remains in the $\varepsilon$-tube. Very often these chattering solutions correspond closely to the real behavior of a system that should in principle strictly follow eqs. (1). For example, in on-off temperature control systems the heating should be on [off] when the temperature is below [above] the desired value, while in practice the heating system remains on [off] untill the temperature reaches a slightly higher [lower] value from below [above]. Obviously, for $\varepsilon$ tending to zero chattering solutions tend toward sliding solutions. Moreover, if $T_{1}(\varepsilon)\left[T_{2}(\varepsilon)\right]$ is the time needed by the chattering solution to cross the $\varepsilon$-tube coming from $S_{1}\left[S_{2}\right]$, then the coefficient $\lambda$ in (2) is the limit for $\varepsilon$ tending to zero of the ratio $T_{1} /\left(T_{1}+T_{2}\right)$.

In accordance with (Gatto et al., 1973), equilibria of (3), where the vectors $f^{(i)}(x)$ are transversal to $\Sigma_{S}$ and anti-collinear, are called pseudo-equilibria of (1) (they are called quasiequilibria in (Filippov, 1988)). This implies that a pseudo-equilibrium $P$ is an internal point of 
a sliding segment. A nonsingular sliding point $x \in \Sigma_{S}$ where $f^{(1)}=0$ is characterized by $\lambda=$ 1 and hence $g=0$. Points of this kind and the analogous ones with $f^{(2)}=0$ (where $\lambda=0$ and $g=0)$ are called boundary equilibria.

A sliding segment terminates either at a boundary equilibrium $X$, or at a point $T$ (called tangent point) where the vectors $f^{(i)}(T)$ are nonzero but one of them is tangent to $\Sigma$.

A tangent point $T$ with $f^{(1)}$ tangent to $\Sigma$ is said to be visible [invisible] if the orbit of $\dot{x}=f^{(1)}(x)$ starting at $T$ belongs initially to $S_{1}\left[S_{2}\right]$, as shown in Fig. 5. A similar definition holds for the vector field $f^{(2)}$.

State portraits of Filippov systems can have multiple sliding segments and be rather complex. Figure 6 shows one example, characterized by

- two sliding segments $\left(T_{1} T_{2}\right.$ and $\left.T_{3} T_{4}\right)$ for a total of four tangent points (three visible and one not);

- two standard equilibria (stable foci $F_{1}$ and $F_{2}$ );

- one unstable standard (i.e., non-sliding and non-crossing) limit cycle $\gamma_{1}$ entirely contained in region $S_{1}$;

- three pseudo-equilibria (two pseudo-saddles $P S_{1}$ and $P S_{2}$, and one stable pseudo-node $P N$ );

- one stable sliding and crossing limit cycle $\gamma_{2}$ (passing through points $T_{1}, A$, and $B$ );

- four attractors (the two equilibria $F_{1}$ and $F_{2}$, the pseudo-equilibrium $P N$, and the sliding cycle $\left.\gamma_{2}\right)$.

The basin of attraction of each attractor is easily identifiable and can involve the stable manifolds of the pseudo-saddles. For example, the basin of attraction of the pseudo-node (shaded region in Fig. 6) is delimited by the stable manifolds of the two pseudo-saddles.

Two Filippov systems of the form (1) are topologically equivalent if there is a homeomorphism $h: R^{2} \rightarrow R^{2}$ that maps the state portrait of one system onto the state portrait of the other, preserving orientation of the orbits. Notice that all sliding segments of one system are mapped onto sliding segments of the other. Moreover, we require that $h$ maps the discontinuity boundary $\Sigma$ of one system onto the discontinuity boundary of the other system. 
If a one-parameter family of Filippov systems is considered (i.e., $f^{(1)}, f^{(2)}$, and $H$ depend also upon a parameter $\alpha \in R$ ), we say that the system exhibits a bifurcation at $\alpha=\alpha_{0}$ if by an arbitrarily small parameter perturbation we get a topologically nonequivalent system.

Of course, a Filippov system can have standard bifurcations, i.e. bifurcations not involving structural changes in the sliding segments. For example, in the case of Fig. 6, the standard cycle $\gamma_{1}$ might shrink when the parameter $\alpha$ is increased and finally collide for $\alpha=\alpha_{0}$ with the stable focus $F_{1}$. This corresponds to a standard subcritical Hopf bifurcation (Kuznetsov, 2004). For $\alpha$ slightly greater than $\alpha_{0}$ the cycle $\gamma_{1}$ does not exist anymore and the focus $F_{1}$ is unstable.

In order to point out which kind of degeneracies are needed to obtain not only standard bifurcations but also sliding bifurcations, i.e. bifurcations involving some structural change in the sliding segments, it is convenient to follow a geometric approach, which consists of detecting the structural changes generated by variations of the parameter $\alpha$ in a special set of trajectories, called characteristic frame. Such a frame is simply composed of

- standard equilibria and non-sliding cycles,

- sliding segments with their pseudo-equilibria and tangent points,

- stable and unstable manifolds of standard saddles and standard manifolds of pseudosaddles,

- trajectories entering $S_{1}$ or $S_{2}$ from tangent points.

For example, the characteristic frame of the system described in Fig. 6 is the set of trajectories shown in Fig. 7. Notice that some of the trajectories emerging from tangent points return to a sliding segment in finite time (see points $B$ and $E$ in Fig. 7), while others do not (trajectory emerging from $T_{2}$ in Fig. 7).

The interest in the characteristic frame is motivated by the fact that all bifurcations correspond to collisions of the various trajectories composing the frame (Kuznetsov et al., 2003). Thus, given the frame corresponding to a particular parameter setting it is very easy to identify by simple inspection all potential collisions that might occur for a perturbation of the parameters and interpret them in terms of qualitative changes of the state portrait of the system. For example, varying a parameter, the two tangent points $T_{3}$ and $T_{4}$ in Fig. 7 might get closer and closer and finally collide. This would correspond to the disappearance of a sliding segment in Fig. 6 which is, indeed, a sliding bifurcation. Another possibility is that 
points $B$ and $P S_{1}$ collide for a particular value of the parameter. This would mark the disappearance of the sliding limit cycle $\gamma_{2}$. In fact, just before the collision of $B$ with $P S_{1}$ the sliding cycle $\gamma_{2}$ exists and has a very long period, because it passes very close to a pseudosaddle (point $P S_{1}$ ) where the sliding motion is very slow. But when the collision occurs the cycle is interrupted because the trajectory tending toward the pseudo-saddle can not pass

through it. This bifurcation is the sliding version of the famous homoclinic bifurcation of standard systems (Kuznetsov, 2004) which is characterized by the coincidence of the unstable and stable manifolds of a saddle. There are many other possible collisions in the characteristic frame that the reader might easily interpret in terms of structural changes of the state portrait in Fig. 6.

Of course, codimension-2 bifurcations are also possible: they simply correspond to a double collision in the characteristic frame. For example, the three pseudo-equilibria $P S_{1}, P N$, and $P S_{2}$ might collide for a specific value of the parameter, thus becoming a unique pseudosaddle. This corresponds to the disappearance of one attractor (the pseudo-node $P N$ ) and is accompanied by the gradual shrinking of its basin of attraction (shaded region in Fig. 6).

The complete list of all sliding bifurcations in second-order Filippov systems is so long that it cannot be presented here. The interested reader might refer to Kuznetsov et al. (2003), where all bifurcations are discussed in detail and the basic aspects of numerical bifurcation analysis are also given. As for the software, the most appropriate reference is Dercole and Kuznetsov (2005).

\section{THE PUZZLE METHOD AND FOUR EXAMPLES}

We propose in this section a method, called puzzle method, for the analysis of secondorder Filippov systems. The method is based on the use of the characteristic frame (see previous section) and is proposed through the analysis of four ecological examples. The aim is to show that the puzzle method is strategically important for carrying out a complete bifurcation analysis.

In each example there are two interconnected populations and only one of them is exploited, unless this is temporarily prohibited. The bifurcation analysis is performed with respect to the exploitation parameter but also with respect to a second control parameter 
interpreting the effort of protecting the populations. Thus, in principle, the obtained results could be used to find reasonable trade-off solutions between exploitation and protection in renewable resources management.

The first example is very simple and could be discussed without explicitly pointing out the puzzle method, which is more formally identified when solving the second and third examples. The strategic value of the method is pointed out in the last example.

\section{Example 1}

Assume that $x_{1}$ and $x_{2}$ are the densities of young and adult individuals of the same population and that only adult individuals reproduce and can be harvested. If aging is density independent and recruitment is increasing but saturating with density, the evolution of young individuals can be described by

$$
\dot{x}_{1}=-\left(a+d_{1}\right) x_{1}+b \frac{x_{2}}{c+x_{2}}
$$

where $a$ and $d_{1}$ are aging and death rate of young individuals and $b$ and $c$ are parameters characterizing the recruitment function. If adult individuals are harvested at constant effort when their density is above a given threshold $P$, and not harvested in the opposite case, then their evolution is described by

$$
\dot{x}_{2}=a x_{1}-d_{2} x_{2} \quad \text { if } x_{2}<P
$$

and

$$
\dot{x}_{2}=a x_{1}-\left(d_{2}+q E\right) x_{2} \quad \text { if } x_{2}>P
$$

where $d_{2}$ is adult death rate, $q$ is catchability coefficient and $E$ is harvesting effort. In the following, we assume that in natural conditions (i.e., for $E=0$ ) the reproductive number of the population, namely the product of the number of progeny produced by an adult in its life $\left(b / c d_{2}\right)$ time the probability a juvenile survives to adulthood $\left(a /\left(a+d_{1}\right)\right)$ is greater than 1 . This condition is equivalent to $a b>c d_{2}\left(a+d_{1}\right)$ and guarantees that the population can persist if not harvested (i.e., the equilibrium $\left(x_{1}, x_{2}\right)=(0,0)$ of $(4,5)$ is unstable). 
System (4-6) is obviously a Filippov system where the boundary $\Sigma$ and the regions $S_{1}$ and $S_{2}$ are

$$
\begin{aligned}
& \Sigma=\left\{\left(x_{1}, x_{2}\right): x_{2}=P\right\} \\
& S_{1}=\left\{\left(x_{1}, x_{2}\right): x_{2}<P\right\} \\
& S_{2}=\left\{\left(x_{1}, x_{2}\right): x_{2}>P\right\}
\end{aligned}
$$

and the state equations are $(4,5)$ in region $S_{1}$ and $(4,6)$ in region $S_{2}$.

If the target is to analyze the behavior of the system for all possible values of the parameters $(E, P)$, the first thing one can do is to find out if sliding is possible. For this, one must check if the components of vector $\dot{x}$ transversal to $\Sigma$ (i.e. $\dot{x}_{2}$ evaluated for $x_{2}=P$ with (5) and (6)) can be of opposite sign, i.e.

$$
\left(a x_{1}-d_{2} P\right)\left(a x_{1}-\left(d_{2}+q E\right) P\right)<0
$$

This inequality is always satisfied on the segment

$$
\frac{d_{2}}{a} P<x_{1}<\frac{d_{2}+q E}{a} P \quad x_{2}=P
$$

which is therefore a sliding segment. Notice that in this case the sliding segment exists and is unique for all parameter values. This sliding segment and its two tangent points $T_{1}=\left(\frac{d_{2}}{a} P\right.$, $P)$ and $T_{2}=\left(\frac{d_{2}+q E}{a} P, P\right)$ are therefore elements of the characteristic frame. Since cycles are not possible in this system (divergence is negative everywhere) the other possible elements of the characteristic frame are equilibria in $S_{1}$ and $S_{2}$, pseudo-equilibria, stable and unstable manifolds of saddles, and trajectories entering $S_{1}$ or $S_{2}$ from tangent points. Let us concentrate first on pseudo-equilibria, which are points on $\Sigma$ where the vectors $\dot{x}$ computed with the state equations holding in $S_{1}$ and $S_{2}$ are anti-collinear. Since, in this case, $\dot{x}_{1}$ is the same in both regions, the pseudo-equilibria must be characterized by $\dot{x}_{1}=0$, i.e., if they exist, they are at the intersection of the sliding segment $T_{1} T_{2}$ with the null-isocline $\dot{x}_{1}=0$. From (4) it follows immediately that there is a unique pseudo-equilibrium whenever its $x_{1}$-coordinate 


$$
x_{1}=\frac{b}{a+d_{1}} \frac{P}{c+P}
$$

is in between the $x_{1}$-coordinates of the tangent points $T_{1}$ and $T_{2}$.

Figure 8 shows the sliding segment $T_{1} T_{2}$ on $\Sigma$, the null-isoclines $\dot{x}_{1}=0$ and $\dot{x}_{2}=0$, and the directions of the vector $\dot{x}$ in the various regions (i.e. the signs of $\dot{x}_{1}$ and $\dot{x}_{2}$ ). The figure corresponds to parameter values (reported in the caption) for which the pseudo-equilibrium is present. The directions of the vector $\dot{x}$ in the various regions show that the pseudoequilibrium is a stable pseudo-node (denoted by $P N$ ) and that points $T_{1}$ and $T_{2}$ are invisible tangent points (so that no trajectory enters $S_{1}$ or $S_{2}$ from them). In conclusion, the characteristic frame is simply the sliding segment with its two tangent points $T_{1}$ and $T_{2}$ and the pseudo-equilibrium $P N$.

Small perturbations of any parameter (not only $E$ and $P$ ) imply, generically, small perturbations of the three points $T_{1}, T_{2}$, and $P N$, so that by simply looking at the characteristic frame one can immediately identify two candidate bifurcations: the collision of $P N$ with $T_{1}$ and the collision of $P N$ with $T_{2}$. Moreover, from eqs. $(7,8)$ one can easily verify that both these bifurcations are, indeed, possible and are characterized by the two following relationships among the parameters

$$
\begin{array}{ll}
P=\frac{a b}{\left(d_{1}+a\right) d_{2}}-c & \left(P N \equiv T_{1}\right) \\
P=\frac{a b}{\left(d_{1}+a\right)\left(d_{2}+q E\right)}-c & \left(P N \equiv T_{2}\right)
\end{array}
$$

Notice that, at these bifurcations, the pseudo-equilibrium actually becomes a standard equilibrium because it is characterized by $\dot{x}_{1}=\dot{x}_{2}=0$. The corresponding bifurcation curves in the parameter space $(E, P)$ identify three regions 1,2 , and 3 as shown in Fig. 9. The two curves merge at a point of the $P$-axis which is a codimension-2 point (collision of $T_{1}, T_{2}$, and $P N$ in the characteristic frame). The null-isoclines and the characteristic frame reported in Fig. 8 are those of region 2. 
In order to understand if we have found all bifurcations, we must iterate our process first by determining the characteristic frames in regions 1 and 3 and then by investigating them in order to see if they suggest new candidate bifurcations. In this case, the process is very simple. Figure 10 shows the characteristic frames (and the null-isoclines) in regions 1 and 3, respectively. The frames are composed of the sliding segment $T_{1} T_{2}$ and of one trajectory entering in $S_{1}$ or $S_{2}$ from one of the tangent points (the visible one) and ending in the standard equilibrium $X$ (intersection of the null-isoclines). Each one of these two characteristic frames suggests two bifurcations, namely the collision of the tangent points $T_{1}$ and $T_{2}$ and the collision of the equilibrium $X$ with $T_{2}$ (in Fig. 10a) or with $T_{1}$ (in Fig. 10b). However, the first bifurcation is not possible, since the $x_{1}$-coordinate of $T_{1}$ is always smaller than that of $T_{2}$. By contrast, the second bifurcation is possible and when the collision occurs the characteristic frame is simply the sliding segment with an equilibrium on one of its two terminal points. But this corresponds exactly to the two bifurcations we have already obtained (see eqs. (9) and (10)), so that we can conclude that the diagram of Fig. 9 is actually complete.

Bifurcation diagrams are usually presented together with the state portraits characterizing each region, so that the final product of a bifurcation study is a diagram of the kind shown in Fig. 11. Once this diagram has been obtained, one can easily compute various indicators of interest for each combination of the parameters. For example, in the present case one has explicit formulas for the values of $\bar{x}_{1}$ and $\bar{x}_{2}$ of the two populations at equilibrium (or at pseudo-equilibrium). In other words, the functions $\bar{x}_{1}(E, P)$ and $\bar{x}_{2}(E, P)$ are known in each region of the parameter space and it is straightforward to verify that, as expected, $\partial \bar{x}_{1,2} / \partial E \leq 0$ and $\partial \bar{x}_{1,2} / \partial P \geq 0$.

Before continuing with other examples, it is worth to remark that the model we have just discussed is extremely simple. Indeed, it has only one attractor and two bifurcations, which are both sliding bifurcations and can be determined analytically.

In the other examples we will have more bifurcations (a mix of sliding and standard bifurcations), that will allow the reader to better perceive the strategic role of the characteristic frame.

\section{Example 2}

Assume that $x_{1}$ and $x_{2}$ are the densities of a logistic prey and a Holling type II predator described, in natural conditions, by the Rosenzweig-MacArthur model (Rosenzweig and 
MacArthur, 1963) (see also McCann and Yodzis (1995) for a detailed discussion of this model and its extensions in the case of food chains composed of vertebrates and invertebrates)

$$
\begin{aligned}
& \dot{x}_{1}=x_{1}\left[r\left(1-\frac{x_{1}}{K}\right)-a \frac{x_{2}}{b+x_{1}}\right] \\
& \dot{x}_{2}=x_{2}\left[c a \frac{x_{1}}{b+x_{1}}-d\right]
\end{aligned}
$$

where $r$ and $K$ are net growth rate and carrying capacity of the prey, and $a, b, c$, and $d$ are maximum predation rate, half saturation constant, efficiency, and death rate of the predator. In the following we assume

$$
a c>d
$$

i.e., predator can grow when prey are abundant, and

$$
K>b \frac{a c+d}{a c-d}
$$

i.e., the attractor is a prey-predator limit cycle which is actually globally stable in the first quadrant (May, 1972; Cheng, 1981; Wrzosek, 1990). Figure 12 shows the null-isoclines $\dot{x}_{1}=$ 0 and $\dot{x}_{2}=0$ (dashed lines) and the state portrait of system (11) when conditions (12) and (13) are satisfied (recall that condition (13) is satisfied when the predator vertical isocline is to the left of the vertex of the parabola $\dot{x}_{1}=0$ ). The characteristic frame is composed of three equilibria (two saddles and an unstable focus), the stable and unstable manifolds of the saddles, and a cycle. Now, assume that predator are harvested at constant effort $E$ and that an alternative prey with constant density $P$ is guaranteed to them in order to overcome critical periods of low abundance of the first prey. Moreover, assume that predator simply feed on the prey that maximizes their fitness $\dot{x}_{2} / x_{2}$ (Genkai-Kato and Yamamura, 1999). Thus, if the predator functional response is $a^{\prime} P /\left(b^{\prime}+P\right)$ when feeding on $P$ and $c^{\prime}$ is the corresponding efficiency, the density $x_{1}^{*}$ at which the predator is indifferent between its two sources of food is 


$$
x_{1}^{*}=\frac{c^{\prime} a^{\prime}}{c a} \frac{b P}{b^{\prime}+\left(1-\frac{c^{\prime} a^{\prime}}{c a}\right) P}
$$

Since we want to consider the case in which the threshold (14) is positive for any value of $P$, we assume

$$
c a>c^{\prime} a^{\prime}
$$

i.e., $x_{1}$ is more profitable than $P$ when abundant. In conclusion, the system is a Filippov system described by

$$
\begin{array}{ll}
\dot{x}_{1}=x_{1}\left[r\left(1-\frac{x_{1}}{K}\right)\right] & \text { if } x_{1}<x_{1}^{*}\left(\text { region } S_{1}\right) \\
\dot{x}_{2}=x_{2}\left[c^{\prime} a^{\prime} \frac{P}{b^{\prime}+P}-d-q E\right] & \text { if } x_{1}>x_{1}^{*}\left(\text { region } S_{2}\right) \\
\dot{x}_{1}=x_{1}\left[r\left(1-\frac{x_{1}}{K}\right)-a \frac{x_{2}}{b+x_{1}}\right] & \\
\dot{x}_{2}=x_{2}\left[c a \frac{x_{1}}{b+x_{1}}-d-q E\right] &
\end{array}
$$

As already said, the parameters $r, K, a, b, c, a^{\prime}, b^{\prime}, c^{\prime}$, and $d$ satisfy inequalities (12), (13), and (15). As far as the control parameters $E$ and $P$ are concerned, we add two more constraints. First we assume that predator cannot survive only on $P$, i.e.

$$
c^{\prime} a^{\prime} \frac{P}{b^{\prime}+P}<q E+d
$$

and second we limit the analysis to the case in which the threshold $x_{1}^{*}($ see (14)) is lower than the prey carrying capacity $K$, i.e. 


$$
\frac{c^{\prime} a^{\prime}}{c a} \frac{b P}{b^{\prime}+\left(1-\frac{c^{\prime} a^{\prime}}{c a}\right) P}<K
$$

The points $(E, P)$ satisfying $(18,19)$ belong to the four regions $1,2,3$, and 4 in the left panel of Fig. 13. The boundaries of the four regions are bifurcation curves and the state portraits in each region are sketched in the smaller panels. We now show how we have produced these bifurcation curves step by step using the characteristic frame.

First of all, it is worth checking if sliding is possible on the boundary $\Sigma$ separating the two regions $S_{1}$ and $S_{2}$ of our Filippov system. Sliding is possible if the transversal components to $\Sigma$ of $\dot{x}$, namely $\dot{x}_{1}$ evaluated for $x_{1}=x_{1}^{*}$ with (16) and (17), can be of opposite sign, i.e. if

$$
\left(1-\frac{x_{1}^{*}}{K}\right)\left[r\left(1-\frac{x_{1}^{*}}{K}\right)-\frac{a x_{2}}{b+x_{1}^{*}}\right]<0
$$

Since we have assumed that $x_{1}^{*}<K$ (see eq. (19)), we can conclude that sliding is always possible provided $x_{2}$ is sufficiently large. More precisely, the sliding segment is identified by

$$
x_{1}=x_{1}^{*} \quad x_{2}>x_{2}^{*}=\frac{r}{a}\left(1-\frac{x_{1}^{*}}{K}\right)\left(b+x_{1}^{*}\right)
$$

with $x_{1}^{*}$ as in (14). A comparison with eq. (11) shows that the sliding segment is actually the part of $\Sigma$ above the prey isocline of system (11) (dashed parabola in Fig. 12).

In order to start the analysis we must first determine the state portrait of system $(16,17)$ for a positive pair $(E, P)$. Since we already know the state portrait for $E=P=0$ (see Fig. 12) we can try to understand how this state portrait changes for very small perturbations of $E$ and $P$. A positive $P$ will simply give rise to a positive threshold $x_{1}^{*}$ and to a sliding segment parallel to the $x_{2}$-axis and above the dashed parabola in Fig. 12. Thus, if $P$ is very small, the sliding segment will be very close to the $x_{2}$-axis. On the other hand, if $E$ is very small, the cycle will be only slightly different from that in Fig. 12 so that, in conclusion, if both $P$ and $E$ are small the sliding segment and the cycle will not interfere, and the state portrait will be as in panel 2 of Fig. 13 (this means that points sufficiently close to the origin in the left panel of Fig. 13 
belong to region 2, though this is not visible at the scale of the figure). Of course, the boundaries of region 2 must correspond to collisions within the characteristic frame which is composed of two saddles, their stable and unstable manifolds (not mentioned in the following), one unstable focus, one stable cycle, one sliding segment with its tangent point, and the trajectory entering $S_{2}$ from it and tending toward the limit cycle. The collisions that the structure of this characteristic frame suggests are only two. One is the standard Hopf bifurcation corresponding to the collision of the cycle with the unstable focus requiring that the cycle shrinks to a point as the parameters vary. The other is the so-called grazing bifurcation corresponding to the collision of the limit cycle with the sliding segment, which can only occur at the tangent point. The Hopf bifurcation occurs when the vertical predator isocline $\left(\dot{x}_{2}=0\right)$ is passing trough the vertex of the prey isocline $\left(\dot{x}_{1}=0\right)$, namely when condition (13) with the equality sign (Hopf condition for system (11)) holds with $d$ replaced by $(d+q E)$, i.e. when

$$
E=E_{H}=\frac{1}{q}\left(a c \frac{K-b}{K+b}-d\right)
$$

By contrast, the grazing bifurcation cannot be determined explicitly, because the preypredator cycle is not known in closed form. The bifurcation curve separating region 2 from region 1 in Fig. 13 must therefore be produced numerically. The most effective way of doing this is to look for all periodic solutions of any period $\tau$ of the two differential equations (17) (which holds in $S_{2}$ ) passing through the tangent point $\left(x_{1}^{*}, x_{2}^{*}\right)$ of the sliding segment (see eq. (20)). This corresponds to find all solutions of the following problem (known as twoboundary value problem)

$$
\begin{aligned}
& \dot{x}_{1}=x_{1}\left[r\left(1-\frac{x_{1}}{K}\right)-a \frac{x_{2}}{b+x_{1}}\right] \\
& \dot{x}_{2}=x_{2}\left[c a \frac{x_{1}}{b+x_{1}}-d-q E\right]
\end{aligned}
$$




$$
\begin{aligned}
& x_{1}(0)=\frac{c^{\prime} a^{\prime}}{c a} \frac{b P}{b^{\prime}+\left(1-\frac{c^{\prime} a^{\prime}}{c a} P\right)} \\
& x_{2}(0)=\frac{r}{a}\left(1-\frac{c^{\prime} a^{\prime}}{K c a} \frac{b P}{b^{\prime}+\left(1-\frac{c^{\prime} a^{\prime}}{c a} P\right)}\right)\left(b+\frac{c^{\prime} a^{\prime}}{c a} \frac{b P}{b^{\prime}+\left(1-\frac{c^{\prime} a^{\prime}}{c a} P\right)}\right) \\
& x_{1}(\tau)=x_{1}(0) \\
& x_{2}(\tau)=x_{2}(0)
\end{aligned}
$$

which has 6 equations and 7 unknowns $\left(E, P, \tau, x_{1}(0), x_{2}(0), x_{1}(\tau), x_{2}(\tau)\right)$. A problem like this has, generically, a one-dimensional family of solutions because the difference between the number of unknowns and the number of equations is 1 . The solutions can be represented as a curve in the seven-dimensional space of the unknowns and the projection of this curve onto the $(E, P)$ space is the grazing bifurcation curve. In practice, the curve in the sevendimensional space is produced through a numerical technique called "continuation" which, starting from a first point obtained through simulation, produces through classical predictioncorrection algorithms a nearby point and then iterates (Kuznetsov, 2004, Chap. 10). Of course, all these computations must be carried out with some specialized software. SlideCont (Dercole and Kuznetsov, 2004; Dercole and Kuznetsov, 2005), which is the most effective software for numerical bifurcation analysis of Filippov systems, produces the grazing bifurcation curve of Fig. 13 in a few seconds.

The bifurcations occurring on the boundaries of region 2 in Fig. 13 allows us to specify uniquely the state portraits of the system on the other side of the same boundaries. In fact, crossing the Hopf bifurcation curve by increasing the effort $E$ one obtains a state portrait like that of region 2 but with the cycle and the unstable focus substituted by a stable focus. Similarly, crossing the grazing bifurcation curve by increasing $P$ one must obtain a state portrait with a sliding cycle.

In order to proceed, one must now look at the two new state portraits, identify the potential collisions that their characteristic frames suggest, and finally check if these collisions can really occur. Let us start with the state portrait in region 1, which has a characteristic frame composed of two saddles, one unstable focus, a sliding segment with one tangent point from which a trajectory enters region $S_{2}$ and returns to the sliding segment to form a sliding cycle. There are two possible collisions one can imagine in such a characteristic frame. One is the 
collision of the point of return on the sliding segment with the tangent point, but this is the grazing bifurcation we have already discussed. The other is the collision of the unstable focus with the tangent point which obviously involves the shrinking of the sliding cycle. This bifurcation is actually impossible inside the region of interest because if the tangent point would be an equilibrium, the predator, which is indifferent at that point between its two food sources, could persist by feeding only on $P$, thus contradicting one of the assumptions we have made (see eq. (18)). Actually, the bifurcation occurs when the parameters satisfy the relationship obtained by replacing the inequality sign in (18) with the equality sign. In other words, the corresponding bifurcation curve is the upper boundary of region 1 . Notice that such a curve merges with the grazing $(G)$ and the Hopf $(H)$ bifurcation curves at a codimension-2 bifurcation point at which the three collisions occur at the same time. In conclusion, the analysis of the state portrait in region 1 does not suggest any new bifurcation.

Finally, the characteristic frame of the state portrait in region 3 suggests three possible bifurcations. One is the Hopf bifurcation that we have already discussed (see eq. (21)), while the two others correspond to the collision of the stable focus with the tangent point or with the saddle on the prey axis. The first one again yields the upper boundary of region 3 . By contrast, the collision of the focus with the saddle on the prey axis (called transcritical bifurcation) is a standard bifurcation in Rosenzweig-MacArthur models (Hsu et al., 1978). It can be explicitly determined by annihilating the predator growth rate in (17) for $x_{1}=K$, thus obtaining

$$
E=E_{T C}=\frac{1}{q}\left(a c \frac{K}{K+b}-d\right)
$$

In order to find out if we have detected all bifurcations in the region of interest, it remains to check if the structure of the characteristic frame of the state portrait in region 4 suggests any new potential collision among its elements. This is, indeed, the case, since in principle we could imagine a collision of the tangent point of the sliding segment with the equilibrium on the prey axis. However, this would imply that the threshold $x_{1}^{*}$ coincides with the prey carrying capacity $K$, a condition that we have explicitly ruled out (see eq. (19)). We can therefore conclude that in the region of interest there are only three bifurcations: a grazing bifurcation of a limit cycle $(G)$, a Hopf bifurcation $(H)$, and a transcritical bifurcation $(T C)$. 
Moreover, the boundaries of the region of interest are themselves bifurcations involving the collision of standard equilibria (focus or saddle) with the tangent point of the sliding segment.

It is worth noticing that the effectiveness of the method of analysis based on the characteristic frame is absolutely independent on the choice of the starting point. In the previous discussion we have decided to start from a point very close to the origin, i.e. from region 2. Then, from the analysis of the characteristic frame in such a region, we have discovered regions 1 and 3 and, finally, looking at the characteristic frame in region 3 we have discovered region 4 . However, we could have started from any other point, for example, by producing through simulation the state portrait of the system for a pair $(E, P)$ in region 3 . Then, from the analysis of the corresponding characteristic frame we would have discovered the Hopf $(H)$ and the transcitical (TC) bifurcation (i.e., regions 2 and 4) and, finally, from the analysis of the characteristic frame in region 2 we would have discovered the grazing bifurcation $(G)$ and region 1. In other words, independently upon the starting point, the method proceeds iteratively by discovering new regions of the bifurcation diagram adjacent to the already discovered ones. In this sense it recalls the most common strategy used for completing a jigsaw puzzle and has been called for this reason "puzzle method".

Once the full bifurcation diagram is available one can draw general conclusions on the behavior of the system and on the impact of the control parameters. For example, in the present case one can observe that the density $P$ of the alternative prey has absolutely no impact on the long term behavior of the system in regions 2, 3, and 4 because in those regions the attractor of the system is to the right of the boundary $\Sigma$ where the dynamics are described by eq. (17) which does not depend on $P$. Thus, an increase of $P$ which might be intuitively proposed to protect populations from risky situations (i.e. too low densities) is actually effective only in region 1 where the attractor is a sliding cycle with minimum values of $x_{1}$ and $x_{2}$ increasing with $P$.

\section{Example 3}

Consider again a prey $\left(x_{1}\right)$ - predator $\left(x_{2}\right)$ assembly described, in the absence of exploitation, by the Rosenzweig-MacArthur model (11) and assume that predator can grow when prey are abundant (see condition (12)) and that the habitat is so rich to guarantee that the prey carrying capacity is above the threshold value (13). Under these conditions, the attractor is a globally stable limit cycle, as shown in Fig. 12. Moreover, assume that the predator population can be exploited at constant effort $E$ (as in the previous example) and that 
in order to avoid risky conditions for the prey, a second habitat, less rich than the natural one, is made available to them where they are better protected from the attacks of the predator (Křivan, 1998). In order to keep the number of control parameters at a minimum value we assume that in the poor but safe habitat the net growth rate of the prey, its carrying capacity and the maximum predation rate are reduced of the same factor $P>1$. Thus, if the criterion used by the prey in selecting the habitat is fitness maximization, the predator density $x_{2}^{*}$ at which prey are indifferent between the two habitats is characterized by the same $\dot{x}_{1} / x_{1}$, i.e.

$$
r\left(1-\frac{x_{1}}{K}\right)-a \frac{x_{2}^{*}}{b+x_{1}}=\frac{r}{P}\left(1-\frac{P x_{1}}{K}\right)-\frac{a}{P} \frac{x_{2}^{*}}{b+x_{1}}
$$

which can be easily solved with respect to $x_{2}^{*}$, thus giving

$$
x_{2}^{*}=\frac{r}{a}\left(b+x_{1}\right)
$$

The straight line (23) is the boundary $\Sigma$ separating the two regions $S_{1}$ and $S_{2}$ of the Filippov system described by

$$
\begin{array}{ll}
\dot{x}_{1}=x_{1}\left[r\left(1-\frac{x_{1}}{K}\right)-a \frac{x_{2}}{b+x_{2}}\right] & \text { if } x_{2}<x_{2}^{*}\left(\text { region } S_{1}\right) \\
\dot{x}_{2}=x_{2}\left[c a \frac{x_{1}}{b+x_{1}}-d-q E\right] & \text { if } x_{2}>x_{2}^{*}\left(\text { region } S_{2}\right) \\
\dot{x}_{1}=x_{1}\left[\frac{r}{P}\left(1-\frac{P x_{1}}{K}\right)-\frac{a}{P} \frac{x_{2}}{b+x_{2}}\right] & \\
\dot{x}_{2}=x_{2}\left[c \frac{a}{P} \frac{x_{1}}{b+x_{1}}-d-q E\right] &
\end{array}
$$

Notice that the boundary $\Sigma$ is independent upon both control parameters $E$ and $P$, while the dynamics depend only upon exploitation $E$ in $S_{1}$, but also upon $P$ in $S_{2}$. 
In order to find if sliding is possible on $\Sigma$, we must check if the transversal components to $\Sigma$ of the vector $\dot{x}$ evaluated with (24) and (25) can be of opposite sign. This corresponds to detect the sign of $\left(-\dot{x}_{1}+(a / r) \dot{x}_{2}\right)$ evaluated with (24) and (25). Straightforward computations allow one to conclude that for any value of $P>1$ there exists a sliding segment $T_{1} T_{2}$, that the motion along this segment is from the right to the left, so that pseudo-equilibria cannot exist, and that the left tangent point (say $T_{1}$ ) is always visible, while the right one (say $T_{2}$ ) is invisible. All state portraits of Fig. 14 point out these properties. Moreover, the sliding segment $T_{1} T_{2}$ shrinks for $P$ tending to 1 since sliding makes no sense when the two habitats are the same. This means that the E-axis of Fig. 14 is a sliding bifurcation (collision of $T_{1}$ and $T_{2}$ ) corresponding to the disappearance of a sliding segment.

In order to perform the bifurcation analysis of system $(24,25)$ we use the puzzle method starting from points close to the $E$-axis where we already know (see previous example with $P$ $=0$ ) that there are two standard bifurcations, namely a Hopf bifurcation $H$ (see eq. (21)) and a transcritical bifurcation $T C$ (see eq. (22)). If $P>1$ and $E>E_{T C}$ the state portrait is that of panel 1 in Fig. 14 where all trajectories tend to point $(K, 0)$ (predator extinction) and some of them slide on the segment $T_{1} T_{2}$. If $P$ is increased and $E$ remains constant, the trajectories below the boundary $\Sigma$ do not vary and the tangent points of the sliding segments remain separated. Thus, the only bifurcation one can imagine by looking at the characteristic frame of panel 1 is the transcritical bifurcation after which the state portrait is like in panel 2. By contrast, the characteristic frame of panel 2 suggests three new bifurcations: collision of the standard focus with $T_{1}$ or $T_{2}$ and Hopf bifurcation. But the first two bifurcations cannot occur since no equilibrium can be on $\Sigma$ where $\dot{x}_{1}$ is always strictly negative (the prey isocline is always below $\Sigma$ and touches it at $x_{1}=0$, see eq. (23-25)). Thus, only the Hopf bifurcation is possible and the new characteristic frame in region 3 suggests only one new bifurcation, namely a grazing of the limit cycle at point $T_{1}$ (grazing at point $T_{2}$ is impossible because $T_{2}$ is an invisible tangent point). Such a bifurcation, denoted by $G$ in Fig. 14, can only be obtained numerically as discussed in the previous example. After this bifurcation the cycle becomes a sliding cycle as shown in panel 4 , and the corresponding characteristic frame suggests only one new bifurcation characterized by the collision of $T_{2}$ with the point $R$ of return on the sliding set $\Sigma_{S}$ of the trajectory entering $S_{1}$ from $T_{1}$. After this bifurcation (see panel 5) the sliding cycle has changed its topology because the sliding segment $T_{2} R$ is now outside the 
cycle and not inside, as in panel 4. The bifurcation separating region 4 from region 5, called buckling bifurcation $(B)$ can be obtained through the solution of a two-boundary value problem imposing $R=T_{2}$. Finally, the characteristic frame of panel 5 suggests only one possible bifurcation, namely the collision of the point of return $R$ with the tangent point $T_{1}$. After this bifurcation, the cycle is not sliding anymore but it crosses twice the boundary $\Sigma$ : For this reason, both the cycle and the bifurcation are called crossing $(C)$. Since no other collision is possible in the characteristic frame of panel 6 , we can conclude that the puzzle method has allowed us to obtain the complete bifurcation diagram of the system.

\section{Example 4}

Consider a prey $\left(x_{1}\right)$ - predator $\left(x_{2}\right)$ assembly and assume that the predator can be harvested when sufficiently abundant. The corresponding Filippov model is as follows:

$$
\begin{array}{ll}
\dot{x}_{1}=x_{1}\left[r\left(1-\frac{x_{1}}{K}\right)-a \frac{x_{2}}{b+x_{2}}\right] & \text { if } x_{2}<P\left(\text { region } S_{1}\right) \\
\dot{x}_{2}=x_{2}\left[c a \frac{x_{1}}{b+x_{1}}-d\right] & \text { if } x_{2}>P\left(\text { region } S_{2}\right) \\
\dot{x}_{1}=x_{1}\left[r\left(1-\frac{x_{1}}{K}\right)-a \frac{x_{2}}{b+x_{2}}\right] & \\
\dot{x}_{2}=x_{2}\left[c a \frac{x_{1}}{b+x_{1}}-d-q E\right] &
\end{array}
$$

It is easy to check that sliding is possible on $\Sigma$ (the horizontal straight line $x_{2}=P$ ) and that the sliding segment can contain one or two pseudo-equilibria. The model looks quite similar to those described in Examples 1-3 but, in reality, it is much more complex. Indeed, in the previous examples, the bifurcations were only 2,3 , and 5 , while they are now 28 as shown in the bifurcation diagram of Fig. 15, which is characterized by 32 regions. The state portraits in each region are not shown, but they can easily be imagined by looking at the characteristic frames sketched in Fig. 16.

The diagrams of Fig. 15 and 16 have been obtained by systematically using the puzzle method. As repeatedly pointed out, one can start the analysis from any point in parameter 
space. Suppose we start from a particular point $(E, P)$ and produce the state portrait through simulation, thus discovering the corresponding characteristic frame. If point $(E, P)$ is, for example, in region 13 of Fig. 15, the characteristic frame we discover is the one reported in panel 13 of Fig. 16. The potential collisions one can imagine within this characteristic frame are five. First, one can imagine a Hopf bifurcation of the standard cycle above $\Sigma$, i.e. the collision of a stable focus with a shrinking cycle. Indeed, this bifurcation is possible at the right boundary of region 13 in Fig. 15 so that the characteristic frame in region 14 is the one sketched in Fig. 16. The second collision suggested by the characteristic frame 13 is the grazing of the standard cycle at the right tangent point of the sliding segment. Also this collision is possible and the corresponding bifurcation curve, produced by solving a twoboundary value problem, is the boundary separating region 13 from region 12 where the characteristic frame is like in Fig. 16. A third possible bifurcation corresponds to the contemporary collision of the lower focus and the pseudo-saddle with the left tangent point of the sliding segment. Such a collision entrains the disappearance (through shrinking) of the sliding cycle below $\Sigma$, as shown by the characteristic frame 19 in Fig. 16. The fourth bifurcation is suggested by the possible collision of the pseudo-saddle with the point of return on the sliding segment of the trajectory entering $S_{1}$ from the left tangent point. Technically speaking, at this bifurcation the unstable manifold of the pseudo-saddle coincides with its stable manifold and this is why this bifurcation has been called (Kuznetsov et al., 2003) sliding homoclinic to a pseudo-saddle. After this bifurcation, the sliding cycle does not exist anymore, as shown in panel 9 of Fig. 16. Finally, there is a fifth bifurcation that the characteristic frame 13 suggests, namely the grazing of the sliding cycle at the left tangent point of the sliding segment. After this bifurcation, the state portrait would contain two standard cycles, one entirely below and one entirely above the boundary $\Sigma$, i.e. one satisfying eqs. (26) and the other eqs. (27). But in a Rosenzweig-MacArthur model the maximum and minimum predator densities along the cycle must, respectively, be above and below the vertex of the prey isocline, as shown in Fig. 12. However, this isocline is the same in eqs. (26) and (27) so that two non-sliding cycles, one above and one below $\Sigma$, cannot exist. In other words, the fifth bifurcation suggested by the characteristic frame 13 cannot occur, so that, in conclusion, region 13 in Fig. 15 is delimited by four boundaries. At this point the puzzle method suggests to apply the same procedure to regions 9, 12, 14, and 19 and then iterate to the newly discovered adjacent regions and continue like so until the bifurcation diagram is completed. It is fair to say, however, that in practice the work does not always proceed as 
smoothly as it might be imagined from the present discussion. Serious obstacles might be found in the numerical computation of some bifurcation curves, as well as in the analysis of the possible collisions within some characteristic frame. For example, the derivation of the bifurcation diagram in Fig. 15 has involved two of us full time for about one month.

This example is much more complex than the previous ones not only because it has more bifurcations but also for three other reasons. The first one, pointed out by Fig. 16, is that there can be multiple attractors. Indeed, for many parameter combinations (see regions 5, 12-15, $22-25,29,31)$, the system has two attractors: at least one of them is a cycle and at least one of them involves sliding. This is a rather unexpected result because when the predator is not protected (i.e. when $P=0$ ) the Rosenzweig-MacArthur model has a single attractor. This means that the protection of an exploited population through an on-off harvesting strategy might give rise to long term dynamics depending upon initial conditions, or, saying the same thing in different words, it might transform a robust system into a system particularly sensitive to impulsive disturbances (which can force the state of the system to switch from one basin of attraction to another).

A second interesting feature of this example is that there are homoclinic bifurcations (see, e.g., the curve separating region 7 from region 8 and the already discussed transition from region 13 to region 9) which are associated with the disappearance of a cycle through the degeneration of its period, which becomes infinitely long when approaching the bifurcation. This phenomenon cannot be present in standard Rosenzweig-MacArthur models but has been proved to be possible in tritrophic food chain models (Kuznetsov et al., 1995; McCann and Yodzis, 1995; Kuznetsov and Rinaldi, 1996; De Feo and Rinaldi, 1998; Boer et al., 1999; Kuznetsov et al., 2001). This means that the introduction of an on-off harvesting strategy in a ditrophic food chain is as powerful as the introduction of a top-predator in producing very long cycles.

Finally, a third important characteristic of this example is that there are catastrophic bifurcations, namely macroscopic transitions in state space due to microscopic variations of the parameters. This important phenomenon is clearly detectable from Fig. 15 and 16. For example, if point $(E, P)$ is in region 5 , but very close to the boundary with region 4 , and the system is at the pseudo-node, a small increase of the threshold $P$ will force the system to enter in region 4 where the only attractor is a sliding cycle. Thus, a small variation in $P$ generates a big surprise, namely a macroscopic transition from a pseudo-equilibrium to a radically different sliding cycle. Moreover, the surprise is even bigger if one notices that a slight 
increase of $P$, which should intuitively protect the predator population, actually gives rise to the opposite effect since the sliding cycle in region 4 is below the threshold $x_{2}=P$.

\section{CONCLUDING REMARKS}

We have summarized in this paper the main properties of Filippov systems (Filippov, 1964; Filippov, 1988) which are systems described by different ordinary differential equations in various regions of state space. The main characteristic of Filippov systems is that they can evolve by sliding along the boundaries separating the various regions. Such systems have already been used in ecology (Charnov, 1976; Stephens and Krebs, 1986; Křivan, 1996; Křivan, 1997a, 1997b; Křivan and Sirot, 1997; Sirot and Křivan, 1997; Křivan, 1998; Boukal and Křivan, 1999; Genkai-Kato and Yamamura, 1999; Křivan and Sikder, 1999; Van Baalen et al., 2001; Dercole et al., 2003; Křivan, 2003; Křivan and Eisner, 2003; Křivan and Schmitz, 2003; Křivan and Diehl, 2005; Meza et al., 2005; Srinivasu and Gayatri, 2005; Costa and Meza, 2006; Dercole et al., 2006; Schreiber et al., 2006), in particular for describing cases in which individuals of one population are fitness-maximizers and therefore switch between alternative habitats or diets as soon as one population becomes too scarce or too abundant. The attention has been focused on bifurcations (Arnold, 1982), in particular on sliding bifurcations, but the analysis has been restricted to second order systems, i.e. systems with only two populations, because only for this case the theory of sliding bifurcations is complete (Kuznetsov et al., 2003). A general method, called "puzzle method" has been proposed for organizing the bifurcation analysis of the system. The method works for any kind of system but is particularly effective for the analysis of Filippov systems, where the bifurcations can be really many. The key idea of the method is to start from a particular point in parameter space and to extract from its state portrait, produced through simulation, a special set of trajectories, called characteristic frame, from which it is easy to detect, through simple inspection, all bifurcations that might potentially occur for small parameter variations. Then, in a second phase, which very often requires the use of specialized software (Dercole and Kuznetsov, 2004; Dercole and Kuznetsov, 2005), one can find out which are the bifurcations that really occur in the system. A by-product of this analysis is a series of new characteristic frames of regions of parameter space close to the starting point. Then, the process is repeated for each one of these characteristic frames, and new characteristic frames characterizing adjacent 
regions are produced. Thus, provided the number of bifurcations is finite, the complete bifurcation diagram is produced step-by-step by expanding around already detected regions, i.e. around already composed pieces of the puzzle.

Four ecological examples have been presented in order to illustrate the proposed method. They all deal with population communities which are at the same time exploited and protected. The bifurcation analysis is not always simple and in some cases (Example 4) the puzzle method turns out to be essential for producing a complete bifurcation diagram. Although this was not the target of the paper, the results obtained through the analysis of the four examples show that the introduction of on-off exploitation strategies can transform a simple system, like the Rosenzweig-MacArthur ditrophic food chain, into a very complex system with multiple attractors, homoclinic bifurcations and catastrophes.

We hope that this paper can popularize Filippov systems among ecologists and that the puzzle method we have suggested will be used to analyze various models. A possible interesting application concerns the coevolution of prey-predator communities characterized by fast and slow dynamics. In such a case, in fact, the coevolution of the two phenotypic traits (one for the prey and one for the predator) turns out to be described, under suitable assumptions, by a Filippov system, which has been mainly analyzed through simulation (Dercole et al., 2006). A complete bifurcation analysis of such a system seems to be possible through the systematic use of the puzzle method and could hopefully give interesting contributions to the theory of prey-predator coevolution (Abrams, 2000). Many other applications are also possible, in particular in the field of renewable resources management.

Of course in many, if not all, applications that one would naturally consider of interest, the number of populations involved is greater than 2. In these cases the puzzle method can still be used but there might be conceptual difficulties associated with the definition and interpretation of the characteristic frame. This is an important issue that we leave open with the hope it will attract the attention of mathematical biologists. 


\section{FIGURE CAPTIONS}

Fig. 1 On the boundary $\Sigma$ the orbit is crossing (a) if the transversal components to $\Sigma$ of $f^{(1)}$ and $f^{(2)}$ have the same sign, or sliding (b) if the transversal components of $f^{(1)}$ and $f^{(2)}$ are of opposite sign.

Fig. 2 Four different state portraits of a Filippov system (see Example 2).

Fig. 3 Filippov rule: the vector $g(x)$ tangent to $\Sigma_{S}$ is a convex combination of $f^{(1)}(x)$ and $f^{(2)}(x)$.

Fig. 4 Trajectories in $S_{1}$ and $S_{2}$ (a), and chattering solutions (b) in an $\varepsilon$-tube around $\Sigma$.

Fig. 5 Visible (a) and invisible (b) tangent point. The thick orbit is a sliding orbit.

Fig. 6 State portrait of a Filippov system with two sliding segments $\left(T_{1} T_{2}\right.$ and $\left.T_{3} T_{4}\right)$, two standard equilibria (two stable foci $F_{1}$ and $F_{2}$ ), three pseudo-equilibria (two pseudosaddles $P S_{1}$ and $P S_{2}$ and a stable pseudo-node $P N$ ), and two limit cycles (one unstable and standard $\left(\gamma_{1}\right)$ and one stable and sliding-crossing $\left.\left(\gamma_{2}=T_{1} A B T_{1}\right)\right)$. The shaded region is the basin of attraction of the pseudo-node.

Fig. 7 Characteristic frame of the system described in Fig. 6.

Fig. 8 Null-isoclines $\dot{x}_{1}=0$ and $\dot{x}_{2}=0$, sliding segment $T_{1} T_{2}$ (with invisible tangent points) and pseudo-node $P N$ of system (4-6) for the following parameter setting: $a=c=d_{1}=$ $d_{2}=q=E=1, b=3, P=0.3$.

Fig. 9 Bifurcation curves of system (4-6) in the space $(E, P)$ for the parameter setting specified in Fig. 8. 
Fig. 10 Null-isoclines, sliding segment $T_{1} T_{2}$ and standard equilibrium $X$ for system (4-6) for two different pairs $(E, P)$. (a): $E=0.2, P=0.13$, i.e. $(E, P)$ is in region 1 of Fig. 9 . (b): $E=0.5, P=0.8$, so that $(E, P)$ is in region 3 of Fig. 9. All other parameters are at their reference values (see caption of Fig. 8). The characteristic frames (solid lines) contain also a trajectory entering in $S_{1}$ or $S_{2}$ from the visible tangent point and ending in the standard equilibrium $X$.

Fig. 11 Complete bifurcation diagram of system (4-6) composed of bifurcation curves and state portraits characterizing each region of parameter space.

Fig. 12 State portrait of the Rosenzweig-MacArthur system (11) for $r=K=a=1, b=0.2, c$ $=0.1, d=0.06$. The characteristic frame is composed of three equilibria (two saddles and an unstable focus) and one stable limit cycle.

Fig. 13 Bifurcation diagram of model $(16,17)$ for the following parameter setting: $r=q=1$, $K=1.5, a=a^{\prime}=5 / 3, b=2 / 3, c=0.4, d=0.1, b^{\prime}=4 / 3, c^{\prime}=0.3$. Curves $G, H$, and TC are grazing, Hopf, and transcritical bifurcations, respectively.

Fig. 14 Bifurcation diagram of model $(24,25)$ for the following parameter setting: $r=5, K=$ $q=1, a=2, b=0.4, c=0.7, d=0.1$ (notice on the left panel that $1 / P$ linearly scales from 1 to 0 on the vertical axis). Curves $H$ and TC are Hopf and transcritical bifurcations given by (21) and (22). Curves $G, B$, and $C$ are sliding bifurcations, known as grazing, buckling and crossing, respectively.

Fig. 15 Bifurcation curves of model $(26,27)$ for the following parameter setting: $r=K=c=$ $q=1, a=0.3556, b=1 / 3, d=0.0444$. The two grey regions are stretched and magnified in the two side panels.

Fig. 16 Sketch of the characteristic frames in regions 1, 2, ., 32 of Fig. 15. 


\section{REFERENCES}

Arnold, V.I., 1982 Geometrical Methods in the Theory of Ordinary Differential Equations. Springer Verlag, New York.

Abrams, P.A., 2000. The evolution of predator-prey interactions: Theory and evidence. Ann. Rev. Ecology Systematics 31, 79-105.

Boer, M., Kooi, B., Kooijman, S., 1999. Homoclinic and heteroclinic orbits in a tri-trophic food chain. Matth. Biol. 39, 19-38.

Boukal, D.S., Křivan, V., 1999. Lyapunov functions for Lotka-Volterra predator-prey models with optimal foraging behaviour. J. Math. Biol. 39, 493-517.

Brogliato, B., 1999. Nonsmooth Mechanics, second ed. Springer, Berlin.

Broucke, M., Pugh, C.C., Simic, S.N., 2001. Structural stability of piecewise smooth systems. Comput. Appl. Math. 20 (1-2), 51-90.

Charnov, E.L., 1976. Optimal foraging: attack strategy of a mantid. Am. Nat. 101, 141-151.

Chau, N.P., 2000. Destabilising effect of period harvest on population dynamics. Ecol. Model. 127, 1-9.

Cheng, K.S., 1981. Uniqueness of a limit cycle for a predator-prey system. SIAM J. Math. Anal. 12, 541-548.

Costa, M.I.S., Meza, M.E.M., 2006. Application of a threshold policy in the management of multispecies fisheries and predator culling. Math. Med. Biol. 23, 63-75.

Davis, L.S., Johnson, K.N., 1987. Forest Management. McGraw-Hill, New York.

De Feo, O., Rinaldi, S., 1998. Singular homoclinic bifurcations in tri-trophic food chains. Math. Biosci. 148, 7-20.

Dercole, F., Gragnani, A., Kuznetsov, Yu.A., Rinaldi, S., 2003. Numerical sliding bifurcation analysis: An application to a relay control system. IEEE Trans. Circuits Systems I Fund. Theory Appl. 50, 1058-1063. 
Dercole, F., Kuznetsov, Yu.A., 2004. User guide to slidecont 2.0. Department of Mathematics, Utrecht University, The Netherlands http://www.math.uu.nl/people/kuznet/cm/slidecont.pdf.

Dercole, F., Kuznetsov, Yu.A., 2005. SlideCont: An auto97 driver for bifurcation analysis of Filippov systems. ACM Trans. Math. Soft. 31, 95-119.

Dercole, F., Gragnani, A., Ferrière, R., Rinaldi, S., 2006. Coevolution of slow-fast populations: An application to prey-predator systems. Proc. R. Soc. Lond. B. 273, 983990.

di Bernardo, M., Champneys, A., Budd, C., 1998a. Grazing, skipping and sliding: analysis of the nonsmooth dynamics of the DC/DC buck converter. Nonlinearity 11, 858-890.

di Bernardo, M., Garofalo, F., Glielmo, L., Vasca, F., 1998b. Switchings, bifurcations and chaos in DC/DC converters. IEEE Trans. Circuits Systems I Fund. Theory Appl. 45, 133141.

di Bernardo, M., Feigin, M.I., Hogan, S., Homer, M., 1999. Local analysis of C-bifurcations in $n$-dimensional piecewise smooth dynamical systems. Chaos, Sol. Fract. 10, 1881-1908.

di Bernardo, M., Budd, C., Champneys, A., 2001. Unified framework for the analysis of grazing and border-collision in piecewise-smooth systems. Phys. Rev. Lett. 86, 25542556.

di Bernardo, M., Kowalczyk, P., Nordmark, A., 2002. Bifurcations of dynamical systems with sliding: Derivation of normal-form mappings. Physica D 11, 175-205.

di Bernardo, M., Kowalczyk, P., Nordmark, A., 2003. Sliding bifurcations: A novel mechanism for the sudden onset of chaos in dry-friction oscillators. Int. J. Bif. Chaos 13, 2935-2948.

Doedel, E., Kernévez, J., 1986. AUTO: Software for continuation problems in ordinary differential equations with applications. Applied Mathematics, California Institute of Technology, Pasadena, CA

Doedel, E., Champneys, A., Fairgrieve, T., Kuznetsov, Yu.A., Sandstede, B., Wang, X., 1997. AUTO97: Continuation and Bifurcation Software for Ordinary Differential Equations (with HomCont), User's Guide. Concordia University, Montreal, Canada. 
Feigin, M.I., 1994. Forced Oscillations in Systems with Discontinuous Nonlinearities. Nauka, Moscow. In Russian.

Filippov, A.F., 1964. Differential equations with discontinuous right-hand side. In American Mathematical Society Translations, Series 2. AMS, Ann Arbor, 199--231.

Filippov, A.F., 1988. Differential Equations with Discontinuous Right-Hand Sides. Kluwer Academic, Dordrecht.

Fredericksen, T.S., 1998. Limitations of low-intensity selective logging for sustainable tropical forestry. Commun. For. Rev. 77, 262-266.

Funasaki, E., Kot, M., 1993. Invasion and chaos in a periodically pulsed mass-action chemostat. Theor. Popul. Biol. 44, 203-224.

Gatto, M., Mandrioli, D., Rinaldi, S., 1973. Pseudoequilibrium in dynamical systems. Int. J. Syst. Sci. 4, 809-824.

Genkai-Kato, M., Yamamura, N., 1999. Unpalatable prey resolves the paradox of enrichment. Proc. R. Soc. Lond. B. 266, 1215-1219.

Geritz, S.A.H., Kisdi, E., 2004. On the mechanistic underpinning of discrete-time population models with complex dynamics. J. Theor. Biol. 228, 261-269.

Grant, W.E., Pedersen, E.K., Marin, S.L., 1997. Ecology and Natural Resource Management: System Analysis and Simulation. Wiley, New York.

Guckenheimer, J., Holmes, P., 1986. Nonlinear Oscillations, Dynamical Systems and Bifurcations of Vector Fields. Springer Verlag, New York.

Hilborn, R., Walters, C., 1992. Quantitative Fisheries Stock Assessment: Choice, Dynamics and Uncertainty. Chapman and Hall.

Hsu, S.B., Hubbel, S.P., Waltman, P., 1978. A contribution to the theory of competing predators. Ecol. Monogr. 48, 337-349.

Ives, A.R., Gross, K., Jansen, V.A.A., 2000. Periodic mortality events in predator-prey systems. Ecology 81, 3330-3340. 
Křivan, V., 1996. Optimal foraging and predator-prey dynamics. Theor. Popul. Biol. 49, 265290.

Krrivan, V., 1997a. Dynamical consequences of optimal host feeding on host-parasitoid population dynamics. Bull. Math. Biol. 59(5), 809-831.

Krrivan, V., 1997b. Dynamic ideal free distribution: effects of optimal path choice on predator-prey dynamics. Am. Nat. 149(1), 164-178.

Křivan, V., 1998. Effects of optimal antipredator behaviour of prey on predator-prey dynamics: The role of refuges. Theor. Popul. Biol. 53, 131-142.

Krrivan, V., 2003. Competitive co-existence caused by adaptive predators. Evol. Ecol. Res. 5, 1163-1182.

Křivan, V., Sirot, E., 1997. Searching for food or hosts: The influence of parasitoids behaviour on host-parasitoid dynamics. Theor. Popul. Biol. 51, 201-209.

Křivan, V., Sikder, A., 1999. Optimal foraging and predator-prey dynamics, II. Theor. Popul. Biol. 55, 111-126.

Křivan, V., Eisner, J., 2003. Optimal foraging and presator-prey dynamics, III. Theor. Popul. Biol. 63, 269-279.

Křivan, V., Schmitz, O.J., 2003. Adaptive foraging and flexible food web topology. Evol. Ecol. Res. 5, 623-652.

Křivan, V., Diehl, S., 2005. Adaptive omnivory and species coexistence in tri-trophic food webs. Theor. Popul. Biol. 67, 85-99.

Kuznetsov, Yu.A., 2004 Elements of Applied Bifurcation Theory. Springer-Verlag, New York, $3^{\text {rd }}$ ed.

Kuznetsov, Yu.A., Muratori, S., Rinaldi, S., 1995. Homoclinic bifurcations in slow-fast second order systems. Nonlin. Anal. 25, 747-762.

Kuznetsov, Yu.A., Rinaldi, S., 1996. Remarks on food chain dynamics. Math. Biosci. 134, 133. 
Kuznetsov, Yu.A., de Feo, O., Rinaldi, S., 2001 Belyakov homoclinic bifurcations in a tritrophic food chain model: SIAM J. Appl. Math. 62(2), 462-487.

Kuznetsov, Yu.A., Rinaldi, S., Gragnani, A., 2003. One-parameter bifurcations in planar Filippov systems. Int. J. Bif. Chaos 13(8), 2157-2188.

Lakmeche, A., Arino, O., 2001. Nonlinear mathematical model of pulsed therapy of heterogeneous tumors. Nonlinear Anal. R. World Appl. 2, 455-465.

Liu, X., Chen, L., 2003. Complex dynamics of Holling type II Lotka-Volterra predator-prey system with impulsive perturbations on the predator. Chaos Solitons Fract. 16(2), 311320.

Maggi, S., Rinaldi, S., 2006. A second-order impact model for forest fire regimes. Theor. Popul. Biol. 70, 174-182.

May, R.M., 1972. Limit cycles in predator-prey communities. Science 177, 900-902.

McCann, K., Yodzis, P.,1995. Bifurcation structure of a three-species food-chain model. Theor. Popul. Biol. 48, 93-125

Meza, M.E.M., Bhaya, A., Kaszkurewicz, E., da Silveira Costa, M.I., 2005. Threshold policies control for predator-prey systems using a control Liapunov function approach. Theor. Popul. Biol. 67, 273-284.

Reluga, T.C., 2004. Analysis of periodic growth disturbance models. Theor. Popul. Biol. 66, 151-161.

Rosenzweig, M.P., MacArthur, R.H., 1963. Graphical representation and stability conditions of predator-prey interaction. Am. Nat. 97, 209-223.

Schreiber, S.J., Lipcius, R.N., Seitz, R.D., Long, W.C., 2006. Dancing between the devil and deep blue sea: The stabilizing effect of enemy-free and victimless sinks. Oikos 113, 6781.

Shulgin, B., Stone, L., Agur, Z., 1998. Pulse vaccination strategy in the SIR epidemic model. Bull. Math. Biol. 60, 1-26.

Sirot, E., Křivan, V., 1997. Adaptive superparasitism and host-parasitoid dynamics. Bull. Math. Biol. 59(1), 23-41. 
Srinivasu, P.D.N., Gayatri, I.L., 2005. Influence of prey reserve capacity on predator-prey dynamics. Ecol. Model. 181, 191-202.

Stephens, D.W., Krebs, J.R., 1986. Foraging Theory. Princeton University Press, Princeton, New Jersey, USA.

Van Baalen, M., Krrivan, V., Van Rijn, P.C.J., Sabelis, M.W., 2001. Alternative food, switching predators, and the persistence of predator-prey systems. Am. Nat. 157(5), 512524.

Wrzosek, D.M., 1990. Limit cycles in predator-prey models. Math. Biosc. 98, 1-12. 


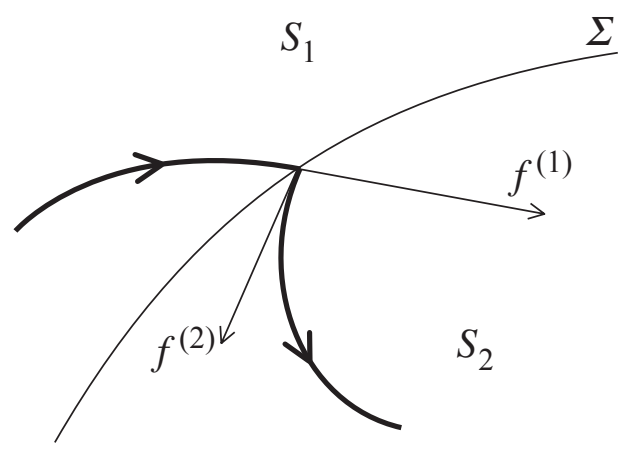

(a)

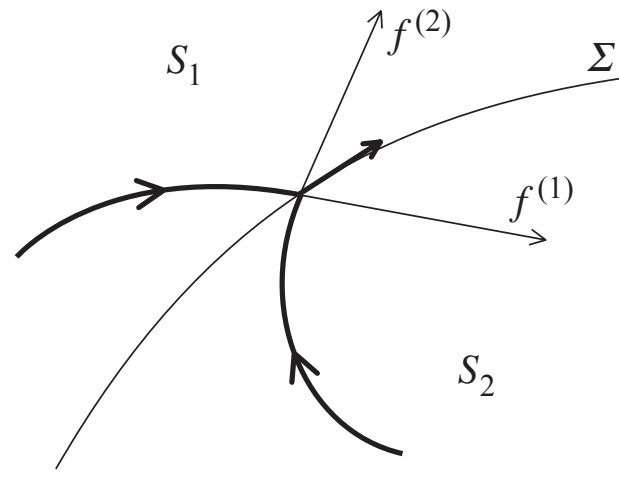

(b)

Figure 1 


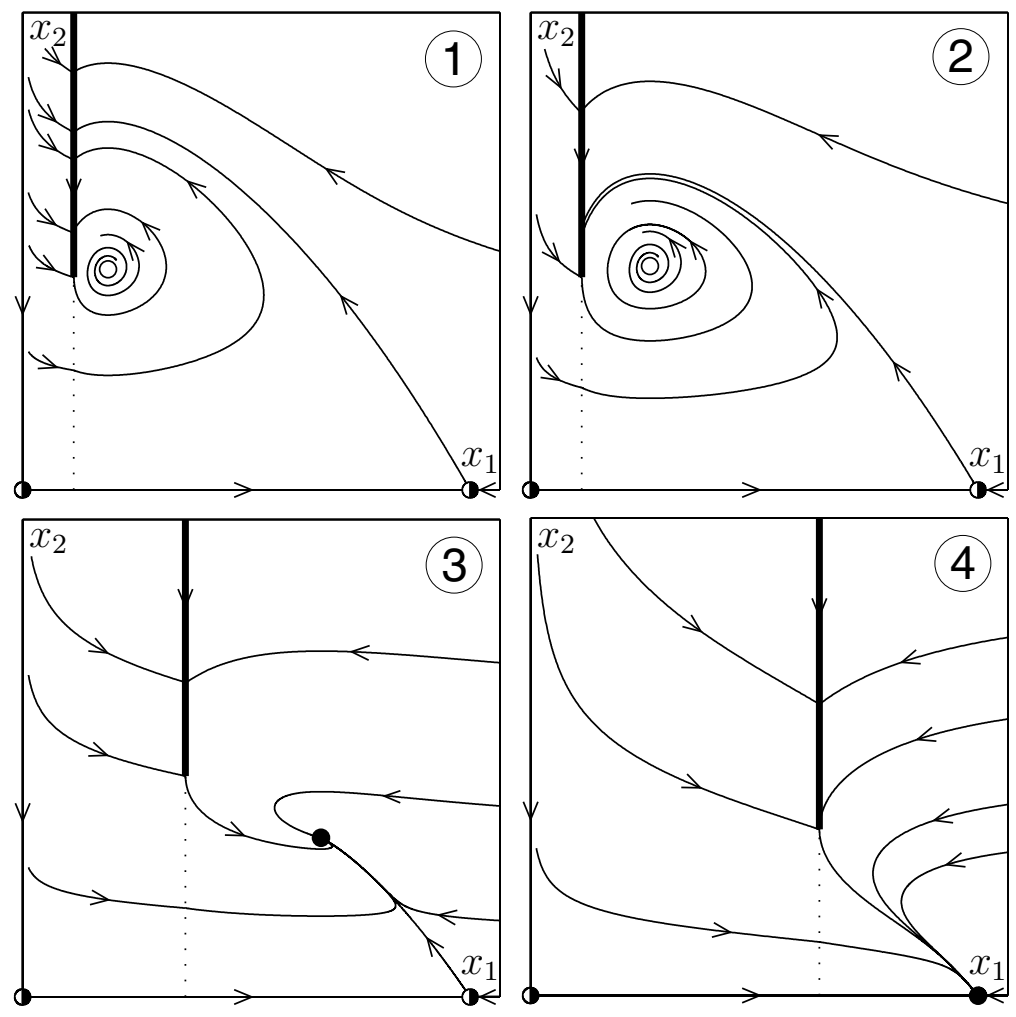

Figure 2 


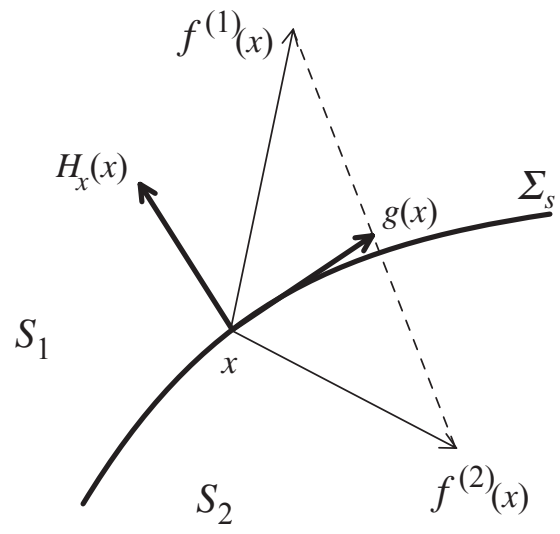

Figure 3 


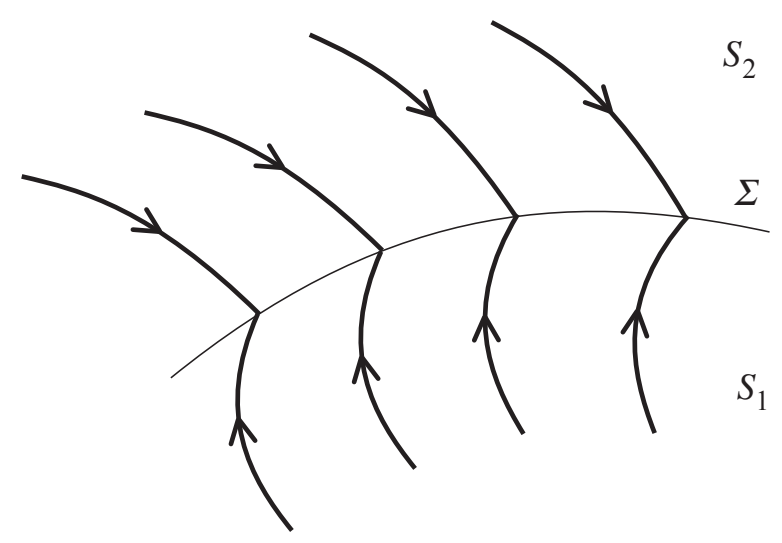

(a)

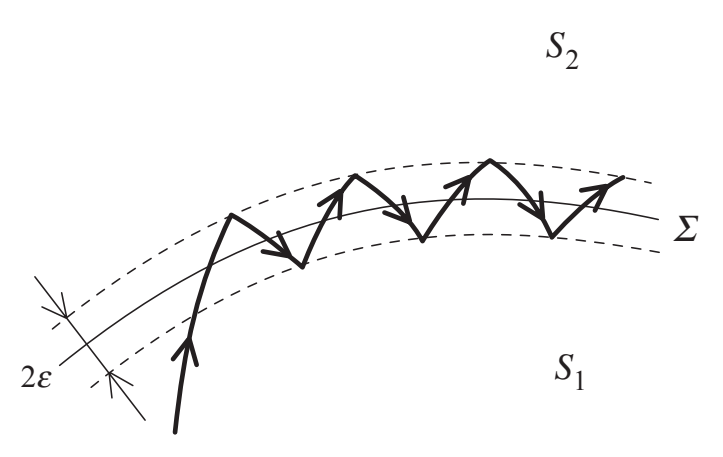

(b)

Figure 4 


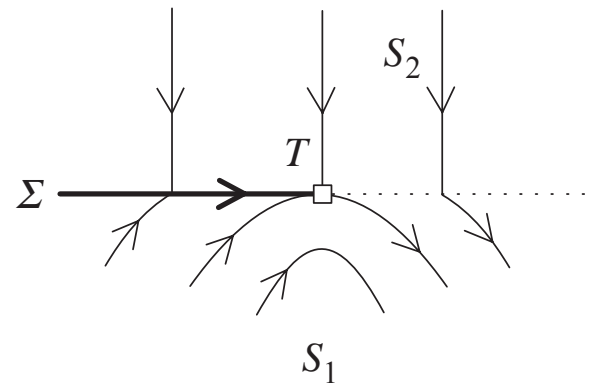

(a)

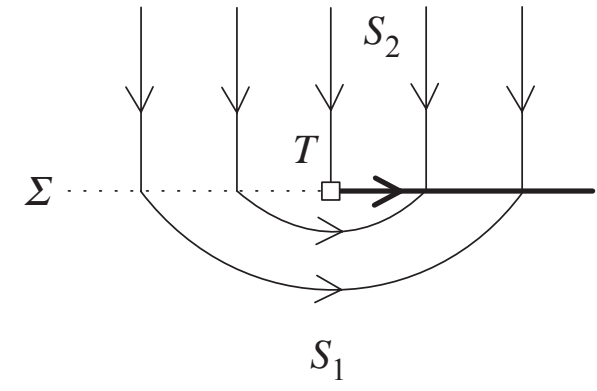

(b)

Figure 5 


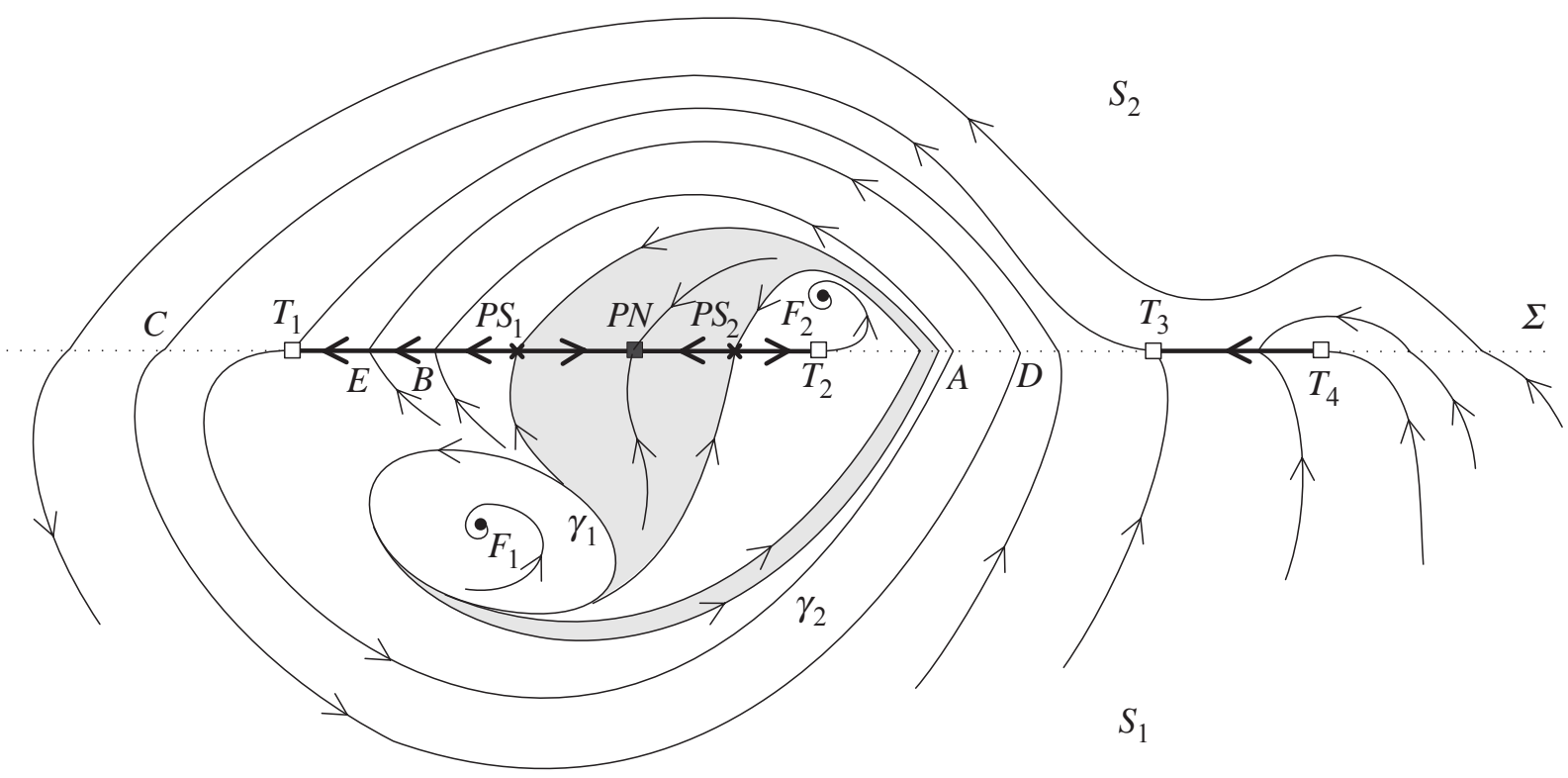

Figure 6 


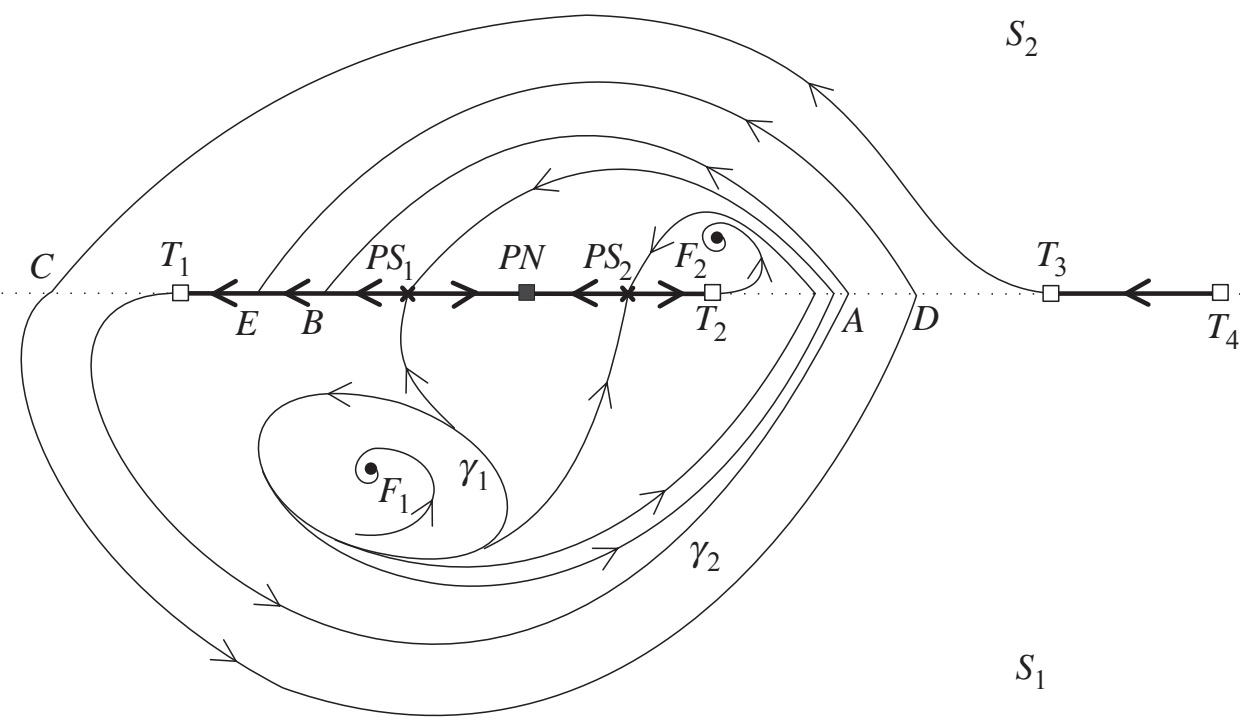

Figure 7 


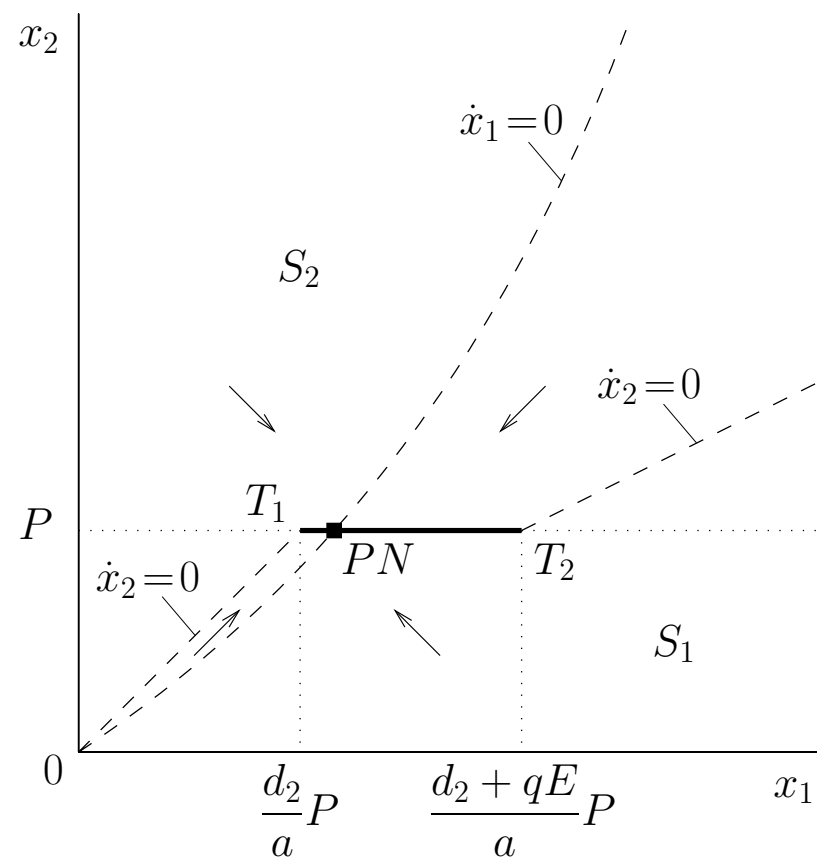

Figure 8 


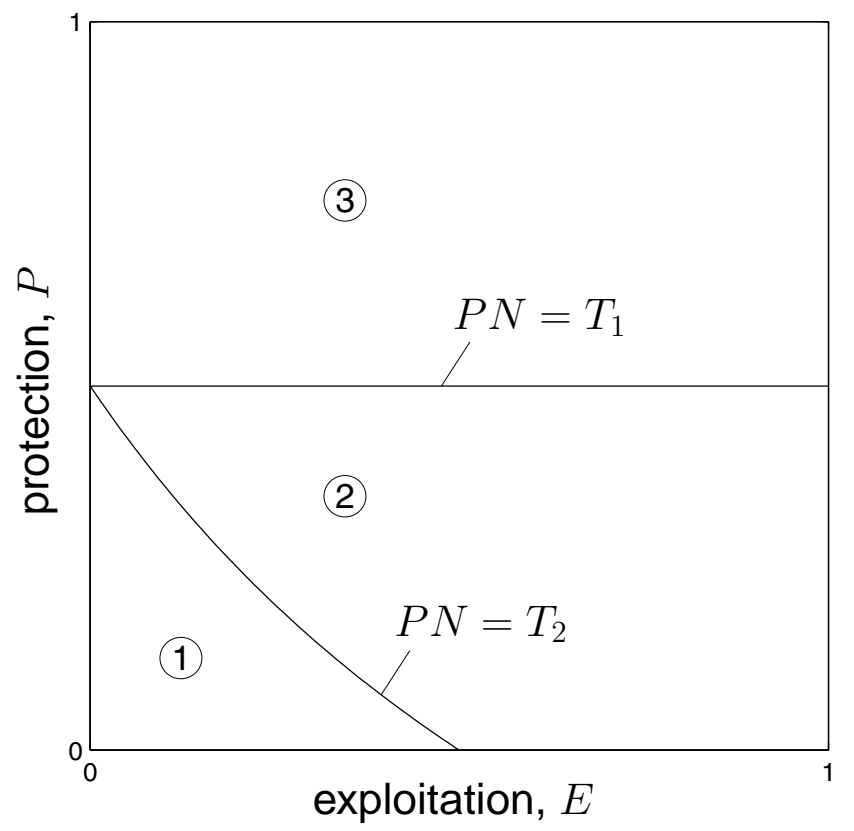

Figure 9 


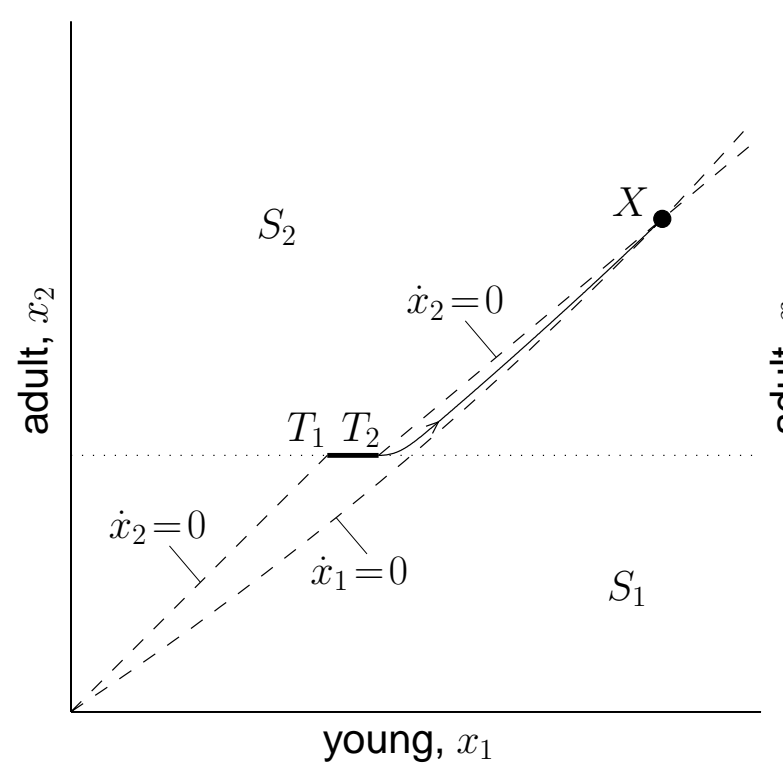

(a)

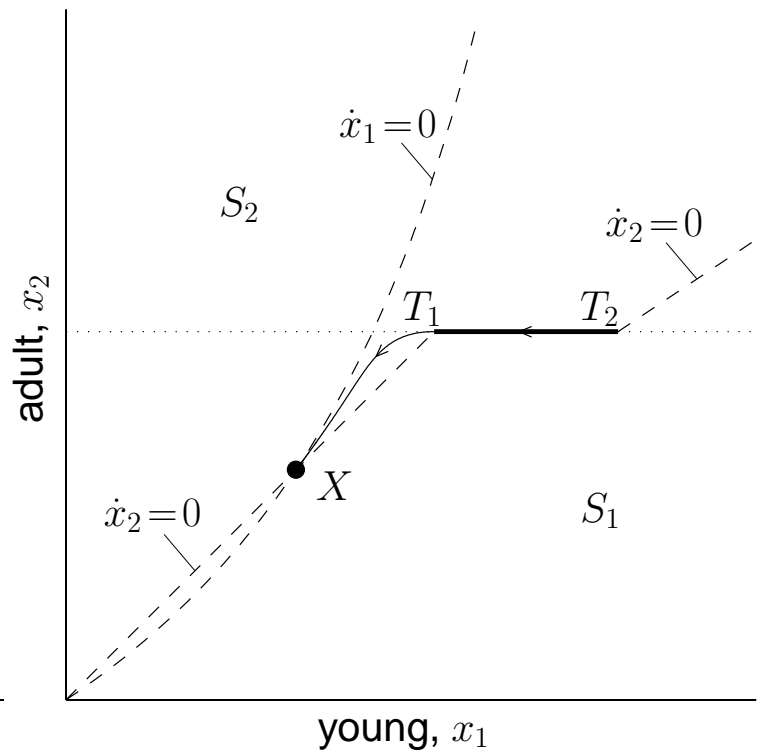

(b)

Figure 10 


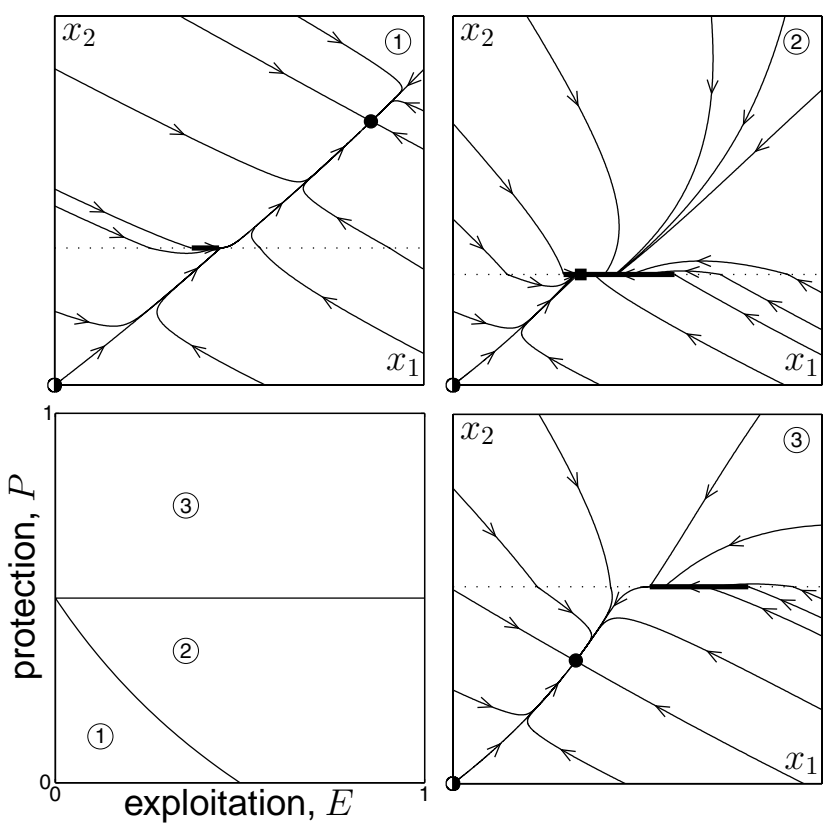

Figure 11 


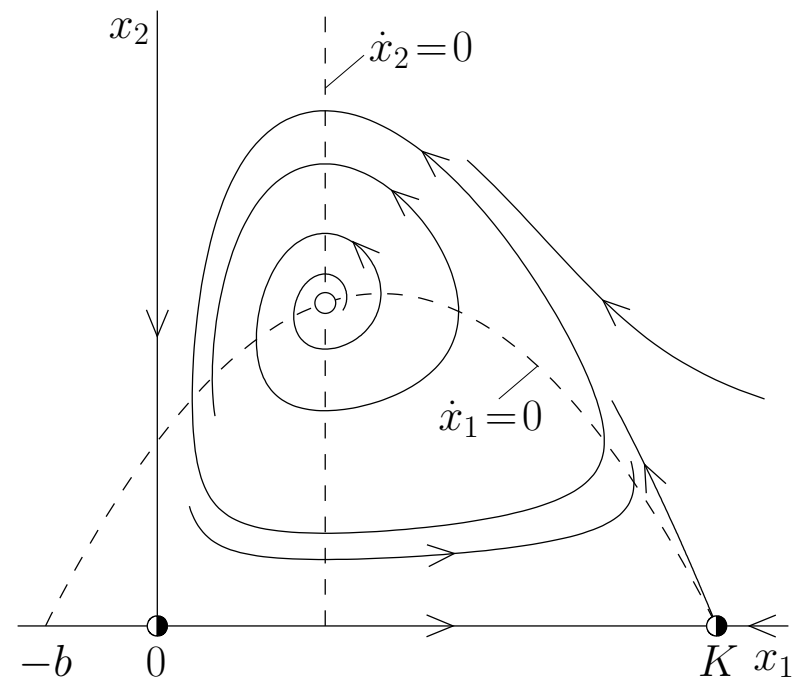

Figure 12 


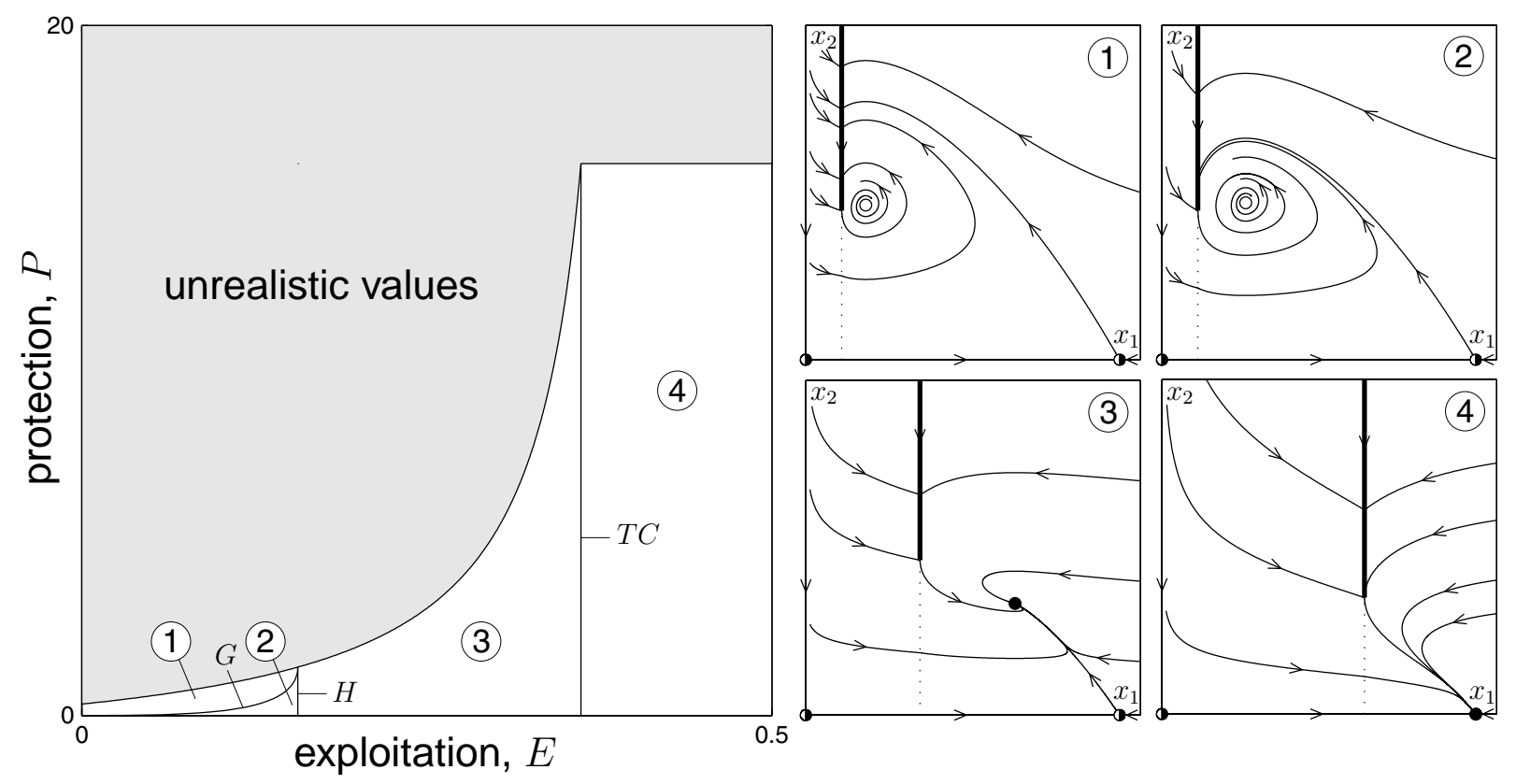

Figure 13 


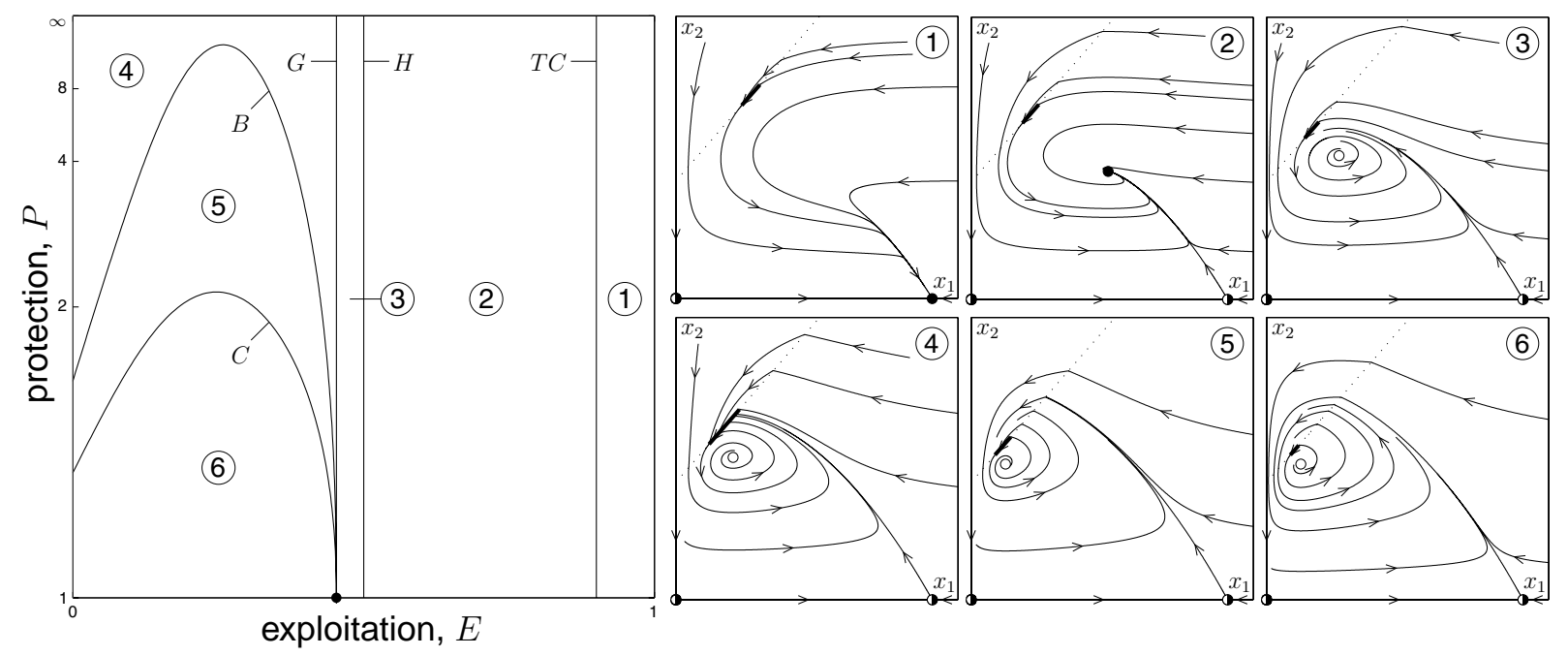

Figure 14 

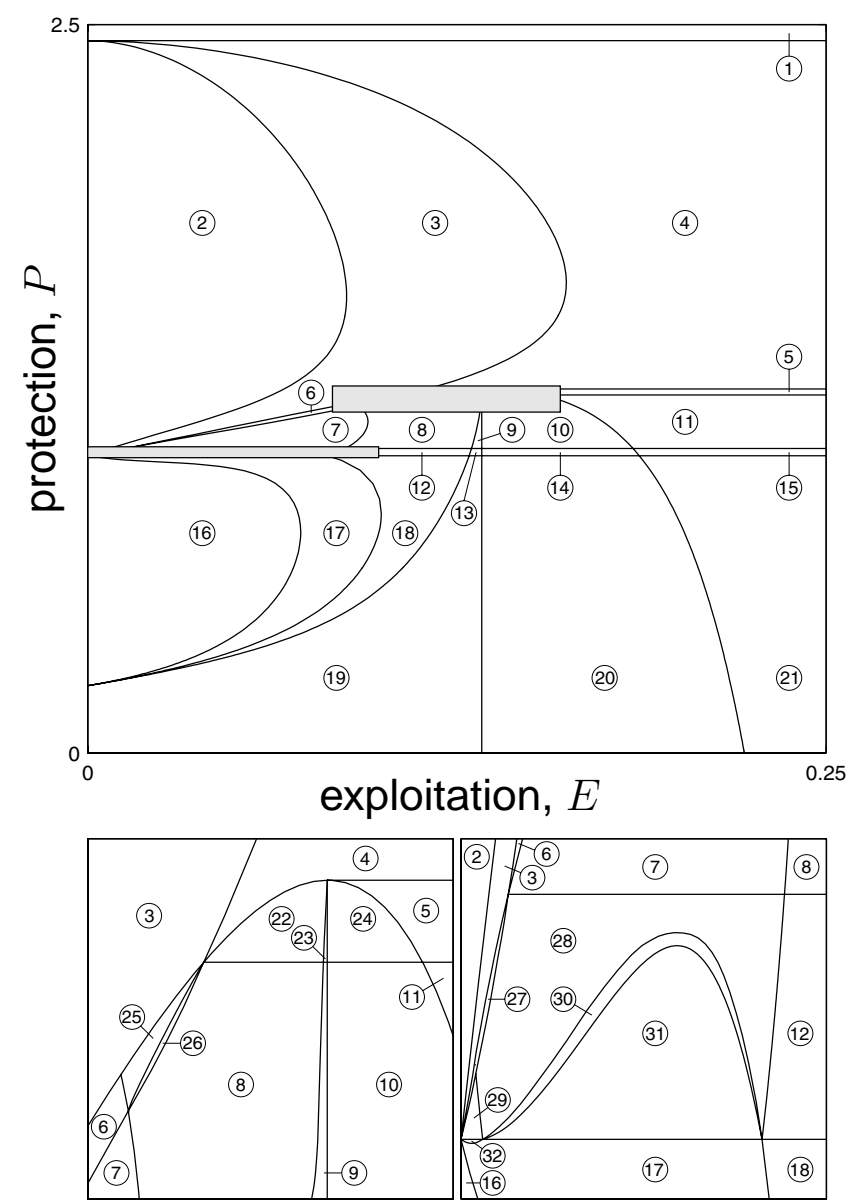

Figure 15 


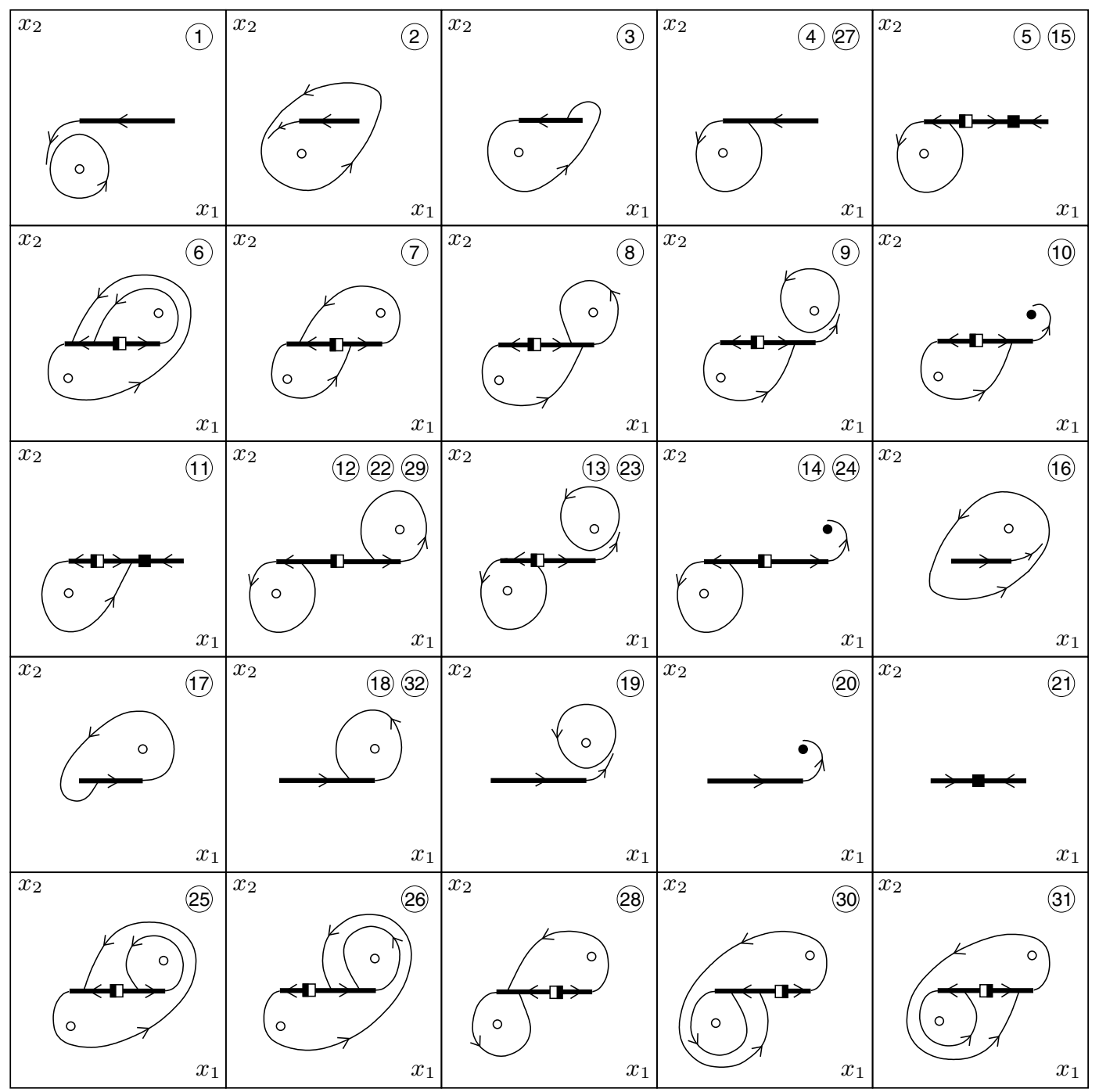

Figure 16 